\title{
Interactions between Light Waves in a Nonlinear Dielectric*
}

\author{
J. A. Armstrong, N. Bloembergen, J. Ducuing, $\dagger$ and P. S. Pershan \\ Division of Engineering and Applied Physics, Harvard University, Cambridge, Massachusetts
}

(Received April 16, 1962)

\begin{abstract}
The induced nonlinear electric dipole and higher moments in an atomic system, irradiated simultaneously by two or three light waves, are calculated by quantum-mechanical perturbation theory. Terms quadratic and cubic in the field amplitudes are included. An important permutation symmetry relation for the nonlinear polarizability is derived and its frequency dependence is discussed. The nonlinear microscopic properties are related to an effective macroscopic nonlinear polarization, which may be incorporated into Maxwell's equations for an infinite, homogeneous, anisotropic, nonlinear, dielectric medium. Energy and power relationships are derived for the nonlinear dielectric which correspond to the Manley-Rowe relations in the theory of parametric amplifiers. Explicit solutions are obtained for the coupled amplitude equations, which describe the interaction between a plane light wave and its second harmonic or the interaction between three plane electromagnetic waves, which satisfy the energy relationship $\omega_{3}=\omega_{1}+\omega_{2}$, and the approximate momentum relationship $\mathbf{k}_{3}=\mathbf{k}_{1}+\mathbf{k}_{2}+\Delta \mathbf{k}$. Third-harmonic generation and interaction between more waves is mentioned. Applications of the theory to the dc and microwave Kerr effect, light modulation, harmonic generation, and parametric conversion are discussed.
\end{abstract}

\section{INTRODUCTION}

$T$ $\mathrm{HE}$ interaction between electromagnetic waves and atomic matter was carried out to higher orders of perturbation theory in the early years of modern quantum mechanics. ${ }^{1-3}$ The interest in the absorpton of two or more light quanta and scattering processes, in which three or more light quanta are involved, has recently been revived, ${ }^{4-7}$ because intense light fluxes available from laser sources have made possible the experimental observation of such higher order processes in the laboratory.

1 Franken ${ }^{8}$ and co-workers observed the creation of the second harmonic of light, corresponding to the elementary processes of the annihilation of two light quanta and the creation of one new quantum with twice the energy. Garrett and Kaiser ${ }^{9}$ observed twophoton absorption.

A crude estimate of the light intensity required for observation of these effects can be obtained as follows. The intensity of a higher order scattering process will be smaller than the scattering in the next lower order process by a factor $\left(E_{l} / E_{\text {at }}\right)^{2} \sim\left[e E a /\left(W_{0}-W_{n}\right)\right]^{2}$, if the scattering is due to electric dipole-type transitions. $E_{l}$ is the electric field in the light wave and $E_{\text {at }} \approx 3 \times 10^{8}$ $\mathrm{V} / \mathrm{cm}$ is a measure of the average atomic electric field

\footnotetext{
* This research was supported jointly by the Office of Naval Research, the Signal Corps of the U. S. Army, the U. S. Air Force, and the Advanced Research Projects Agency.

† On leave from Department de Physique Appliquée, Cie Gle de

Telegraphie sans fil (Orsay), France. O.E.E.C. fellow, 1961.

1 P. A. M. Dirac, Proc. Roy. Soc. (London) A114, 143, 710 (1927).

${ }^{2}$ M. Goeppert-Mayer, Ann. Physik 9, 273 (1931).

${ }^{3}$ J. Frenkel, Wave Mechanics, Advanced General Theory (Oxford University Press, New York, 1934), p. $226 \mathrm{ff}$.

${ }^{4}$ Th. Neugebauer, Acta Phys. Acad. Sci. Hung. 10, 221 (1959).

5 D. A. Kleinman, Phys. Rev. 125, 87 (1962)

${ }^{6}$ R. Braunstein, Phys. Rev. 125, 475 (1962)

${ }^{7}$ W. C. Hennenberger, Bull. Am. Phys. Soc. 7, 14 (1962).

${ }^{8}$ P. Franken, A. E. Hill, C. W. Peters, and G. Weinreich, Phys. Rev. Letters 7, 118 (1961).

${ }^{9}$ W. Kaiser and C. G. B. Garrett, Phys. Rev. Letters 7, 229 (1961)
}

acting on valence electrons, $a$ is the atomic radius, and $W_{0}-W_{n}$ is an average excitation energy of the atom. In cases where parity considerations prohibit electric dipole scattering processes, there is an additional factor of $(a / \lambda)^{2} \sim 10^{-7}$ in this ratio.

Since excellent experimental discrimination between multiple photon and lower order processes is possible, nonlinear effects are readily observable for $\left(E_{l} / E_{\mathrm{at}}\right)^{2}$ $\sim 10^{-10}$ or $E_{l}=3 \times 10^{3} \mathrm{~V} / \mathrm{cm}$. This corresponds to a light intensity of $0.25 \mathrm{MW} / \mathrm{cm}^{2}$. Such intensities are available even in unfocused laser beams. A transient ruby laser pulse, operated with a Kerr cell shutter, has been reported $^{10,11}$ to have a peak power of $10 \mathrm{MW}$ in a beam of about $0.5-\mathrm{cm}^{2}$ cross section. Higher densities are obtainable in focused beams. Much higher peak power densities than have so far been reported will be obtainable.

Coherence effects are of paramount importance in the experiments of Franken ${ }^{8}$ and other investigators ${ }^{12-15}$ who have studied the production of light harmonics. The production of such harmonics may increase as the square of the number of scattering centers. Even in a gaseous medium the interest is not so much in the incoherent scattering of individual molecules, but in the coherent effects of an equivalent continuous medium with the average density. The same distinction arises in the linear theory of dispersion. The incoherent Rayleigh scattering gives rise to the blue color of sky, but the coherent scattering leads to an index of refraction of the air.

${ }^{10} \mathrm{R}$. W. Hellwarth, Advances in Quantum Electronics, edited by J. R. Singer (Columbia University Press, New York, 1961), p. 335 .

${ }^{11}$ F. J. McClung, J. Appl. Phys. (to be published).

12 M. Bass, P. A. Franken, A. E. Hill, C. W. Peters, and G. Weinreich, Phys. Rev. Letters 8, 18 (1962).

13 J. A. Giordmaine, Phys. Rev. Letters 8, 19 (1962).

${ }_{14}$ P. D. Maker, R. W. Terhune, M. Nisenoff, and C. M. Savage, Phys. Rev. Letters 8, 21 (1962).

${ }_{15}$ B. Lax, J. G. Mavroides, and D. F. Edwards, Phys. Rev. Letters 8, 166 (1962). 
The published theoretical papers ${ }^{4-7}$ deal adequately with the incoherent processes, such as multiple photon absorption. The usual treatment of elementary scattering processes from an individual molecule leaves the question of coherence between incident and scattered photons unsolved. The optical index of refraction is best derived by a semiclassical method. ${ }^{16}$ After the expectation value of the induced dipole moment of the atomic system has been calculated quantum mechanically, one reverts at the earliest opportunity to the macroscopic polarization and the continuum theory of Maxwell and Lorentz. ${ }^{17,18}$

In this paper we shall adopt a similar course for the nonlinear part of the polarization. In Sec. II quantummechanical expressions for the nonlinear, induced electric-dipole moments are derived to terms quadratic and cubic in the field strength. They are illustrated with the example of the anharmonic oscillator. In Sec. III, a connection between the microscopic nonlinear properties and the macroscopic field quantities is made. Retardation and higher order moments are also discussed. In Sec. IV the nonlinear polarization is incorporated into Maxwell's equations. Explicit solutions to Maxwell's equations in the infinite nonlinear, anisotropic dielectric are given in Secs. V, VI, and VII. They describe the power transfer between a fundamental wave and its second harmonic and between three plane electromagnetic waves, which satisfy the energy relationship for the frequencies $\omega_{3}=\omega_{1}+\omega_{2}$ and approximately satisfy the momentum relationship for wave vectors, $\mathbf{k}_{3}=\mathbf{k}_{1}+\mathbf{k}_{2}+\Delta \mathbf{k}$ with $|\Delta \mathbf{k}| \ll\left|k_{3}\right|$. The solutions may be regarded as a generalization of the traveling wave, parametric amplifier equations. ${ }^{19}$ These follow as a special case, when the power flow in one of the waves (the pump) is very much larger than the other two. In the present paper all three waves are treated on an equal basis. The extension to the third harmonic and the interaction between four waves is given in Sec. VII. Implications of the theory for experimental situations and devices are briefly discussed in Sec. VIII.

Multiple photon absorption and harmonic generation have, of course, been observed previously in the radiofrequency and microwave range of the electromagnetic spectrum. There propagation effects are usually not important, with the exception of the parametric traveling wave amplifier. In the optical region, phase relationships between the waves propagating in the nonlinear medium play a dominant role. This fact becomes of particular interest at the boundary of a nonlinear dielectric. The well-known laws of reflection and refraction of light in a linear medium can be

${ }^{16}$ H. A. Kramers, Quantum Mechanics (North-Holland Publishing Company, Amsterdam, 1957), pp. 482-489.

${ }^{17} \mathrm{H}$. A. Lorentz, The Theory of Electrons (B. G. Teubner, Leipzig, 1909).

${ }^{18}$ L. Rosenfeld, Theory of Electrons (North-Holland Publishing Company, Amsterdam, 1951).

${ }^{19} \mathrm{~W}$. H. Louisell, Coupled Mode and Parametric Electronics (J. Wiley \& Sons, Inc., New York, 1960). extended to a nonlinear medium. The modified laws of Snell and Fresnel for light harmonics will be treated in a separate paper..$^{20}$

\section{QUANTUM-MECHANICAL CALCULATION OF NONLINEAR SOURCE TERMS}

In this section the semiclassical theory of the interaction between radiation and matter, due to Klein, ${ }^{21}$ will be used to describe the nonlinear interaction of several monochromatic waves in a stationary radiation field. Kramers ${ }^{16}$ has discussed in detail how this semiclassical method conveniently leads to a theory of coherent scattering and linear dielectric constant. His method will be extended to higher order in perturbation theory. This will lead in a natural way to the nonlinear dielectric properties of the medium.

\section{A. Terms in $E^{2}$}

The effect of the radiation on the system is to induce changes in the expectation value of its electron current density. This induced current density is then considered to be a source of scattered radiation. For the sake of definiteness, the field will be considered to be a superposition of three harmonic waves whose frequencies satisfy the the relation $\omega_{1}+\omega_{2}=\omega_{3}$. The vector potential of this incident vacuum field is

$$
\mathbf{A}=\sum_{i=1,2,3} \hat{a}_{i} q_{i} \cos \left(\mathbf{k}_{i} \cdot \mathbf{r}-\omega_{i} t+\phi_{i}\right),
$$

where $\hat{a}_{i}$ is a unit polarization vector. The perturbation Hamiltonian is

$$
\mathfrak{F}_{\mathrm{int}}=\sum_{k}\left[-\frac{e}{m c} \mathbf{A}_{k} \cdot \mathbf{p}_{k}+\frac{e^{2}}{2 m c^{2}} \mathbf{A}_{k} \cdot \mathbf{A}_{k}\right],
$$

where the sum is over all electrons $k$. In writing $\mathfrak{H}_{\text {int }}$ in this form we have assumed a Coulomb gauge,

$$
\operatorname{div} \mathbf{A}=0, \quad \mathbf{E}=-\operatorname{Re}[(1 / c)(\partial \mathbf{A} / \partial t)],
$$

which implies that $\mathbf{p}$ and $\mathbf{A}$ commute. Since $\mathbf{p}$ is Hermitian and $\mathbf{A}$ is real, $\mathfrak{H}_{\text {int }}$ is Hermitian. Note, however, that a particular term in the interaction such as $\mathbf{p} \cdot \hat{a} \exp (i \mathbf{k} \cdot \mathbf{r})$ need not be Hermitian.

The wave functions of the unperturbed system are denoted by $\phi_{n}$ and have energy eigenvalues $W_{n}$. For simplicity we assume the $\phi_{n}$ are products of one-electron wave functions $\phi_{n}=\Pi_{n} \phi_{n}(k)$. If no dc magnetic field is present (and spin is ignored), the wave functions $\phi_{n}$ can be assumed real without loss of generality. Denote the perturbed ground-state wave function by $\psi_{g}$. These wave functions are used to calculate the expectation value of the induced electron current density. We have

$$
\begin{aligned}
\sum_{\text {electrons } \mathrm{k}}\left\{\rho \mathbf{v}_{k}\right\}_{\boldsymbol{g}} & \\
& =\sum_{k} \psi_{\boldsymbol{g}}{ }^{*}(k)\left(e \mathbf{p}_{k} / m-e^{2} \mathbf{A}_{k} / m c\right) \psi_{\boldsymbol{\theta}}(k) .
\end{aligned}
$$

${ }^{20} \mathrm{~N}$. Bloembergen and P. S. Pershan, Phys. Rev. (to be published).

${ }^{21}$ O. Klein, Z. Physik 40, 407 (1927). 
The terms in Eq. (2.4), linear in the fields, have been discussed in detail by Kramers, who shows how they lead to the linear dielectric susceptibility of the medium. In this paper interest centers on the terms quadratic (and cubic) in the fields $\mathbf{A}_{i}$. In order to obtain all terms quadratic in the $\mathbf{A}_{i}$ the perturbed wave functions must be calculated to second order in time-dependent perturbation theory. ${ }^{3}$

$$
\begin{aligned}
\psi_{g}(t)=\sum_{n}\left[\delta_{n g}+a_{n}{ }^{(1)}(\mathbf{p} \cdot \mathbf{A})\right. & +a_{n}{ }^{(1)}(\mathbf{A} \cdot \mathbf{A}) \\
& \left.+a_{n}{ }^{(2)}(\mathbf{p} \cdot \mathbf{A})\right] \phi_{n} e^{-i \omega_{n} t} .
\end{aligned}
$$

The notation makes clear that there are terms both linear and quadratic in the $\mathbf{A}_{i}$ in the first-order expansion coefficients.

The terms in Eq. (2.4) quadratic in the $\mathbf{A}_{i}$ can be grouped according to their frequency dependence. Since the incident field contains three different frequencies, the second-order perturbed wave functions will display time dependences at nine different frequencies. One may, for example, inquire about the nonlinear source term at $\omega_{1}$ due to the interaction between fields at $\omega_{3}$ and $\omega_{2}$, or about the source at $2 \omega_{1}$ due to the interaction of the field at $\omega_{1}$ with itself, etc.
We will illustrate the significance of Eq. (2.4) by computing the source term at $\omega_{3}=\omega_{1}+\omega_{2}$ due to the interaction between the waves at $\omega_{1}$ and $\omega_{2}$.

Our interest in the nonlinear source term at $\omega_{3}$ is limited to that part which will contribute coherently to the incident wave at $\omega_{3}$. We evaluate Eq. (2.4) retaining only those terms with time dependence $\exp \left( \pm i \omega_{3} t\right)$ and proportional to $q_{1} q_{2}$.

$$
\left\{\rho \mathbf{v}\left(\omega_{3}\right)\right\}_{g g}=\left\{\rho \mathbf{v}\left(\omega_{3}\right)\right\}_{g g}{ }^{+} e^{i \omega_{3} t}+\left\{\rho \mathbf{v}\left(\omega_{3}\right)\right\}_{g g} e^{-i \omega_{3} t} .
$$

Equation (2.6) may be regarded as defining the quantities $\{\rho \mathbf{v}\}_{g g}{ }^{ \pm}$. The induced current density Eq. (2.6) has contributions from the time derivatives of all multipole moments of the perturbed electron distribution. However, by suitably retarding this current density, i.e., by multiplying the term in Eq. (2.6) proportional to $\exp \left( \pm i \omega_{3} t\right)$ by $\exp \left( \pm i \mathbf{k}_{3} \cdot \mathbf{r}\right)$, and then taking the expectation value of the resulting expression, the source term at $\omega_{3}$ takes on the form of the time derivative of an effective dipole moment, $\mathfrak{B}_{\text {eff }} \mathrm{NL}^{\mathrm{L}}\left(\omega_{3}\right)$.

$$
\mathfrak{B}_{\text {eff }}{ }^{\mathrm{NL}}\left(\omega_{3}\right)=2 \operatorname{Re}\left[\mathfrak{B}_{-}{ }^{\mathrm{NL}}\left(\omega_{3}\right) e^{-i \omega_{3} t}\right] .
$$

The explicit expression for $\mathfrak{B}_{-}{ }^{\mathrm{NL}}\left(\omega_{3}\right) \exp \left(-i \omega_{3} t\right)$ is (for convenience we omit the sum over electrons):

where

$$
\mathfrak{B}_{-}{ }^{\mathrm{NL}}\left(\omega_{3}\right) e^{-i \omega_{3} t}=\left\langle g\left|\left\{\rho \mathbf{v}\left(\omega_{3}\right)\right\}^{-\cdot} \cdot \exp \left(-i \mathbf{k}_{3} \cdot \mathbf{r}\right)\right| g\right\rangle e^{-i \omega_{3} t}=\frac{i q_{1} q_{2}}{\omega_{3}}\left(\frac{e^{3}}{4 m^{3} c^{2} \hbar^{2}}\right) e^{i\left(\phi_{1}+\phi_{2}-\phi_{3}\right)} e^{-i \omega_{3} t+i \phi_{3}}[\mathfrak{A}+\mathfrak{B}],
$$

$$
\begin{aligned}
& \mathfrak{A}=-m \hbar \sum_{j^{\prime}}\left\{\frac{\left(e^{i\left(\mathbf{k}_{2}-\mathbf{k}_{3}\right) \cdot \mathbf{r}} \hat{a}_{2}\right)_{g j^{\prime}}\left(e^{i \mathbf{k}_{1} \cdot \mathbf{r}} \hat{a}_{1} \cdot \mathbf{p}\right)_{j^{\prime} g}}{\omega_{j^{\prime} g}-\omega_{1}}+\frac{\left(e^{i\left(\mathbf{k}_{2}-\mathbf{k}_{3}\right) \cdot \mathrm{r}} \hat{a}_{2}\right)_{j^{\prime} g}\left(e^{-i \mathbf{k}_{1} \cdot \mathrm{r}} \hat{a}_{1} \cdot \mathbf{p}\right)_{j^{\prime} g} *}{\omega_{j^{\prime} g}+\omega_{1}}+\frac{\left(e^{i\left(\mathbf{k}_{1}-\mathbf{k}_{3}\right) \cdot \mathrm{r}} \hat{a}_{1}\right)_{g j^{\prime}}\left(e^{i \mathbf{k}_{2} \cdot \mathrm{r}} \hat{a}_{2} \cdot \mathbf{p}\right)_{j^{\prime} g}}{\omega_{j^{\prime} g}-\omega_{2}}\right. \\
& \left.+\frac{\left(e^{i\left(\mathbf{k}_{1}-\mathbf{k}_{3}\right) \cdot \mathbf{r}} \hat{a}_{1}\right)_{j^{\prime} g}\left(e^{-i \mathbf{k}_{2} \cdot \mathbf{r}} \hat{a}_{2} \cdot \mathbf{p}\right)_{j^{\prime} g} *}{\omega_{j^{\prime} g}+\omega_{2}}+\frac{\frac{1}{2}\left(\hat{a}_{1} \cdot \hat{a}_{2}\right)\left(e^{-i \mathbf{k}_{3} \cdot \mathbf{r}} \mathbf{p}\right)_{g j^{\prime}}\left(e^{i\left(\mathbf{k}_{1}+\mathbf{k}_{2}\right) \cdot \mathbf{r}}\right)_{j^{\prime} g}}{\omega_{j^{\prime} g}-\omega_{3}}+\frac{\frac{1}{2}\left(\hat{a}_{1} \cdot \hat{a}_{2}\right)\left(e^{-i \mathbf{k}_{3} \cdot \mathbf{r}} \mathbf{p}\right)_{j^{\prime} g}\left(e^{-i\left(\mathbf{k}_{1}+\mathbf{k}_{2}\right) \cdot \mathbf{r}}\right)_{j^{\prime} g}{ }^{*}}{\omega_{j^{\prime} g}+\omega_{3}}\right\}
\end{aligned}
$$

and

$$
\begin{aligned}
& \mathfrak{B}=\sum_{i, i^{\prime}}\left\{\frac{\left(e^{-i \mathbf{k}_{3} \cdot \mathbf{r}} \mathbf{p}\right)_{g j^{\prime}}\left(e^{i \mathbf{k}_{1} \cdot \mathbf{r}} \hat{a}_{1} \cdot \mathbf{p}\right)_{j^{\prime} j}\left(e^{i \mathbf{k}_{2} \cdot \mathbf{r}} \hat{a}_{2} \cdot \mathbf{p}\right)_{j g}}{\left(\omega_{j^{\prime} g}-\omega_{3}\right)\left(\omega_{j g}-\omega_{2}\right)}+\frac{\left(e^{-i \mathbf{k} \mathbf{k}_{3} \cdot \mathbf{r}} \mathbf{p}\right)_{j^{\prime} g}\left(e^{-i \mathbf{k}_{1} \cdot \mathbf{r}} \hat{a}_{1} \cdot \mathbf{p}\right)_{j^{\prime} j^{\prime}}\left(e^{-i \mathbf{k} 2 \cdot \mathbf{r}} \hat{a}_{2} \cdot \mathbf{p}\right)_{j g}{ }^{*}}{\left(\omega_{j^{\prime} g}+\omega_{3}\right)\left(\omega_{j g}+\omega_{2}\right)}\right. \\
& +\frac{\left(e^{-i \mathbf{k}_{3} \cdot \mathrm{r}} \mathbf{p}\right)_{g j^{\prime}}\left(e^{i \mathbf{k}_{2} \cdot \mathrm{r}} \hat{a}_{2} \cdot \mathbf{p}\right)_{j^{\prime} j}\left(e^{i \mathbf{k}_{1} \cdot \mathbf{r}} \hat{a}_{1} \cdot \mathbf{p}\right)_{j g}}{\left(\omega_{j^{\prime} g}-\omega_{3}\right)\left(\omega_{j g}-\omega_{1}\right)}+\frac{\left(e^{-i \mathbf{k}_{3} \cdot \mathrm{r}} \mathbf{p}\right)_{j^{\prime} g}\left(e^{-i \mathbf{k}_{2} \cdot \mathrm{r}} \hat{a}_{2} \cdot \mathbf{p}\right)_{j^{\prime} j} *\left(e^{-i \mathbf{k}_{1} \cdot \mathrm{r}} \hat{a}_{1} \cdot \mathbf{p}\right)_{j g}{ }^{*}}{\left(\omega_{j^{\prime} g}+\omega_{3}\right)\left(\omega_{j g}+\omega_{1}\right)} \\
& +\frac{\frac{1}{2}\left(e^{-i \mathbf{k}_{3} \cdot \mathbf{r}}\right)_{j^{\prime} j}\left(e^{-i \mathbf{k}_{1} \cdot \mathbf{r}} \hat{a}_{1} \cdot \mathbf{p}\right)_{j^{\prime} g}{ }^{*}\left(e^{i \mathbf{k}_{2} \cdot \mathrm{r}} \hat{a}_{2} \cdot \mathbf{p}\right)_{j g}}{\left(\omega_{j^{\prime} g}+\omega_{1}\right)\left(\omega_{j g}-\omega_{2}\right)}+\frac{\frac{1}{2}\left(e^{-i \mathbf{k} \mathbf{k}_{3} \cdot \mathbf{r}} \mathbf{p}^{*}\right)_{j^{\prime} j}\left(e^{i \mathbf{k} \mathbf{k}_{1} \cdot \mathbf{r}} \hat{a}_{1} \cdot \mathbf{p}\right)_{j^{\prime} g}\left(e^{-i \mathbf{k} 2 \cdot \mathrm{r}} \hat{a}_{2} \cdot \mathbf{p}\right)_{j g}{ }^{*}}{\left(\omega_{j^{\prime} g}-\omega_{1}\right)\left(\omega_{j g}+\omega_{2}\right)} \\
& \left.+\frac{\frac{1}{2}\left(e^{-i \mathbf{k}_{3} \cdot \mathbf{r}} \mathbf{p}\right)_{j^{\prime} j}\left(e^{i \mathbf{k}_{1} \cdot \mathbf{r}} \hat{a}_{1} \cdot \mathbf{p}\right)_{j g}\left(e^{-i \mathbf{k}_{2} \cdot \mathbf{r}} \hat{a}_{2} \cdot \mathbf{p}\right)_{j^{\prime} g}{ }^{*}}{\left(\omega_{j^{\prime} g}+\omega_{2}\right)\left(\omega_{j g}-\omega_{1}\right)}+\frac{\frac{1}{2}\left(e^{-i \mathbf{k}_{3} \cdot \mathbf{r}} \mathbf{p}^{*}\right)_{j^{\prime} j}\left(e^{-i \mathbf{k}_{1} \cdot \mathrm{r}} \hat{a}_{1} \cdot \mathbf{p}\right)_{j g}{ }^{*}\left(e^{i \mathbf{k}_{2} \cdot \mathrm{r}} \hat{a}_{2} \cdot \mathbf{p}\right)_{j^{\prime} g}}{\left(\omega_{j^{\prime} g}-\omega_{2}\right)\left(\omega_{j g}+\omega_{1}\right)}\right\} .
\end{aligned}
$$

Analogous expressions define the effective nonlinear dipole moments at frequencies $\omega_{1}$ and $\omega_{2}$. The correctness of the definitions in Eqs. (2.7)-(2.10) may be checked by writing down the time-independent part of the third-order perturbed energy $\left(\mathcal{H C}_{\mathrm{int}}\right)_{g g}$, which is cubic in the field strengths $q$. This energy contains, in principle, contributions from all multipole moments of the system.
Each term in Eqs. (2.9) and (2.10) contains at least one matrix element of the form $(\mathbf{A} \cdot \mathbf{p})_{k l}$. The complex exponentials in the expression (2.1) for $\mathbf{A}$ may be expanded in terms of matrix elements of all the multipole moments of the atomic system. If there is a center of symmetry the wave functions $\phi_{j}$ will have welldefined parity. In this case the pure electric-dipole terms (zeroth order in $k$ ) in $\mathfrak{B}_{\text {eff }}{ }^{\mathrm{NL}}$ will vanish. The 
electric quadrupole (and magnetic dipole) terms, linear in $\mathbf{k}$, will, however, give a finite result.

The assumption will now be made that the atomic system lacks an inversion center. In this case the terms in $\mathfrak{P}_{\text {eff }}{ }^{\mathrm{NL}}$ of zeroth order in $\mathbf{k}$ will dominate all others and we may set all factors of $\exp ( \pm i \mathbf{k} \cdot \mathbf{r})$ equal to unity. In this case a great simplification results, since as was shown by Goeppert-Mayer, ${ }^{2}$ the perturbation $\mathfrak{H}_{\text {int }}$ may now be written

$$
\mathfrak{H}_{\text {int }}=-\mathfrak{B} \cdot \mathbf{E}(t),
$$

where $\mathfrak{P}$ is the total electric dipole moment operator of the molecule and $\mathbf{E}(t)$ is the total (real) local electric field. We again consider the case of three waves with frequencies related as before and write the electric field in the form

$$
\mathbf{E}(t)=\sum_{i=1,2,3} \mathbf{E}_{i} \cos \left(\omega_{i} t+\phi_{i}\right) .
$$

We also observe that, since $\mathbf{A}$ now has only diagonal matrix elements we can write $(\rho \mathbf{v})_{\mathrm{op}}=(\partial \mathfrak{P} / \partial t)_{\mathrm{op}}$ and need only calculate $\langle g|\mathfrak{B}| g\rangle$, retaining as before only terms proportional to $E_{i} E_{j}$ and having time dependence at $\omega_{1}, \omega_{2}$, or $\omega_{3}$. Using the same second-order wave functions we find for $\left(\mathfrak{F}_{\alpha}\right)_{g o} \mathrm{NL}$ the following expression.

$$
\begin{aligned}
& \left(\mathfrak{P}_{\alpha}\right)_{g g}=\cos \left(\omega_{1} t+\phi_{3}-\phi_{2}\right) \sum_{j, j^{\prime}}\left\{\mathfrak{P}_{\alpha j^{\prime} j}\left(\mathfrak{P}_{j^{\prime},} \cdot \mathbf{E}_{2}\right)\left(\mathfrak{B}_{j g} \cdot \mathbf{E}_{3}\right) A_{j j^{\prime}}+\mathfrak{P}_{\alpha j g}\left(\mathfrak{P}_{j^{\prime} j} \cdot \mathbf{E}_{2}\right)\left(\mathfrak{P}_{j g} \cdot \mathbf{E}_{3}\right) B_{j j^{\prime}}\right. \\
& \left.+\mathfrak{P}_{\alpha j^{\prime} g}\left(\mathfrak{P}_{j^{\prime} j} \cdot \mathbf{E}_{3}\right)\left(\mathfrak{P}_{j g} \cdot \mathbf{E}_{2}\right) C_{j j^{\prime}}\right\}+\cos \left(\omega_{2} t+\phi_{3}-\phi_{1}\right) \sum_{j, j^{\prime}}\left\{\mathfrak{P}_{\alpha j^{\prime} g}\left(\mathfrak{B}_{j^{\prime} j} \cdot \mathbf{E}_{1}\right)\left(\mathfrak{B}_{j g} \cdot \mathbf{E}_{3}\right) A_{j j^{\prime}}\right. \\
& \left.+\mathfrak{B}_{\alpha^{\prime} j}\left(\mathfrak{B}_{j^{\prime} g} \cdot \mathbf{E}_{1}\right)\left(\mathfrak{B}_{j g} \cdot \mathbf{E}_{3}\right) B_{j j^{\prime}}+\mathfrak{P}_{\alpha j^{\prime} g}\left(\mathfrak{B}_{j^{\prime} j} \cdot \mathbf{E}_{3}\right)\left(\mathfrak{B}_{j g} \cdot \mathbf{E}_{1}\right) C_{j j^{\prime}}\right\} \\
& +\cos \left(\omega_{3} t+\phi_{1}+\phi_{2}\right) \sum_{j, j^{\prime}}\left\{\mathfrak{B}_{\alpha j^{\prime} g}\left(\mathfrak{B}_{j^{\prime}} \cdot \mathbf{E}_{1}\right)\left(\mathfrak{P}_{j g} \cdot \mathbf{E}_{2}\right) A_{j j^{\prime}}+\mathfrak{P}_{\alpha j^{\prime} g}\left(\mathfrak{B}_{j^{\prime} j} \cdot \mathbf{E}_{2}\right)\left(\mathfrak{P}_{j g} \cdot \mathbf{E}_{1}\right) B_{j^{\prime} j}\right. \\
& \left.+\mathfrak{P}_{\alpha j^{\prime} j}\left(\mathfrak{B}_{j^{\prime} g} \cdot \mathbf{E}_{1}\right)\left(\mathfrak{B}_{j g} \cdot \mathbf{E}_{2}\right) C_{j j^{\prime}}\right\} ;
\end{aligned}
$$

where

$$
\begin{aligned}
& A_{j j^{\prime}}=\frac{1}{2 \hbar^{2}}\left[\frac{1}{\left(\omega_{j g}+\omega_{2}\right)\left(\omega_{j^{\prime} g}+\omega_{3}\right)}\right. \\
& B_{j j^{\prime}}=\frac{1}{2 \hbar^{2}}\left[\frac{1}{\frac{1}{\left(\omega_{j g}-\omega_{2}\right)\left(\omega_{j^{\prime} g}-\omega_{3}\right)}}\right], \\
& C_{j j^{\prime}}=\frac{1}{2 \hbar^{2}}\left[\frac{1}{\left(\omega_{j g}+\omega_{1}\right)\left(\omega_{j^{\prime} g}+\omega_{3}\right)}\right. \\
& \left.+\frac{1}{\left(\omega_{j g}+\omega_{1}\right)\left(\omega_{j^{\prime} g}-\omega_{1}\right)\left(\omega_{j^{\prime} g}-\omega_{3}\right)}\right], \\
& \left.\quad+\frac{1}{\left(\omega_{j g}-\omega_{1}\right)\left(\omega_{j^{\prime} g}+\omega_{2}\right)}\right] .
\end{aligned}
$$

Use has been made of the fact that the wave functions are assumed real.

Note that each Fourier component of $\left(\mathfrak{P}_{\alpha}{ }^{\mathrm{NL}}\right)_{g g}$ may be written as a third-order tensor (dimensions $3 \times 9$ ) operating on an unsymmetrical second-order tensor. This second-order tensor is the direct product of the electric fields at two different frequencies.

$$
\begin{aligned}
\mathfrak{P}_{i} \mathrm{NL}\left(\omega_{1}\right)= & \cos \left(\omega_{1} t+\phi_{3}-\phi_{2}\right) \\
& \quad \times \sum_{j, k} \beta_{i j k}\left(\omega_{1}=\omega_{3}-\omega_{2}\right) E_{3 j} E_{2 k}, \\
\mathfrak{P}_{i}^{\mathrm{NL}}\left(\omega_{2}\right)= & \cos \left(\omega_{2} t+\phi_{3}-\phi_{1}\right) \\
& \quad \times \sum_{j, k} \beta_{i j k}\left(\omega_{2}=\omega_{3}-\omega_{1}\right) E_{3 j} E_{1 k}, \\
\mathfrak{P}_{i} \mathrm{NL}\left(\omega_{3}\right)= & \cos \left(\omega_{3} t+\phi_{1}+\phi_{2}\right) \\
& \times \sum_{j, k} \beta_{i, k}\left(\omega_{3}=\omega_{1}+\omega_{2}\right) E_{1 j} E_{2 k} .
\end{aligned}
$$

$$
\begin{aligned}
& \left(\mathfrak{B}_{\alpha}\right)_{g g}=\cos \left(\omega_{1} t+\phi_{2}-\phi_{1}\right) \sum_{j, j^{\prime}}\left\{\mathfrak{B}_{\alpha j^{\prime} g}\left(\mathfrak{B}_{j^{\prime} j} \cdot \mathbf{E}_{1}\right)\left(\mathfrak{B}_{j g} \cdot \mathbf{E}_{2}\right) A_{j, j^{\prime}}^{\prime}+\mathfrak{B}_{\alpha g j^{\prime}}\left(\mathfrak{B}_{j^{\prime} j} \cdot \mathbf{E}_{2}\right)\left(\mathfrak{B}_{j g} \cdot \mathbf{E}_{1}\right) B_{j, j^{\prime}}^{\prime}\right. \\
& \left.+\mathfrak{P}_{\alpha j^{\prime} j}\left(\mathfrak{P}_{j^{\prime} g} \cdot \mathbf{E}_{1}\right)\left(\mathfrak{P}_{j g} \cdot \mathbf{E}_{2}\right) A^{\prime}{ }_{j, j^{\prime}}\right\}+\cos \left(2 \omega_{1} t+2 \phi_{1}\right) \sum_{j, j^{\prime}}\left\{\mathfrak{P}_{\alpha j^{\prime} g}\left(\mathfrak{B}_{j^{\prime} j} \cdot \mathbf{E}_{1}\right)\left(\mathfrak{B}_{j g} \cdot \mathbf{E}_{1}\right) A_{j, j^{\prime}}\right. \\
& \left.+\mathfrak{B}_{\alpha j^{\prime} j}\left(\mathfrak{B}_{j^{\prime} g} \cdot \mathbf{E}_{1}\right)\left(\mathfrak{B}_{j g} \cdot \mathbf{E}_{1}\right)\left(B^{\prime}{ }_{j j^{\prime}} / 2\right)\right\} \text {, }
\end{aligned}
$$


where

$$
\begin{aligned}
& A^{\prime}{ }_{j, j^{\prime}}=\frac{1}{2 \hbar^{2}}\left[\frac{1}{\left(\omega_{j g}+\omega_{2}\right)\left(\omega_{j^{\prime} g}+\omega_{1}\right)}\right.\left.+\frac{1}{\left(\omega_{j g}-\omega_{2}\right)\left(\omega_{j^{\prime} g}-\omega_{1}\right)}\right], \\
& B^{\prime}{ }_{j, j^{\prime}}=\frac{1}{2 \hbar^{2}}\left[\frac{1}{\left(\omega_{j g}+\omega_{1}\right)\left(\omega_{j^{\prime} g}-\omega_{1}\right)}\right. \\
&\left.\quad+\frac{1}{\left(\omega_{j g}-\omega_{2}\right)\left(\omega_{j^{\prime} g}+\omega_{1}\right)}\right] .
\end{aligned}
$$

\section{B. Terms in $E^{3}$}

When the previous calculations are extended to one higher order in perturbation theory one finds nonlinear source terms of third order in the electric fields. For example, an incident wave at $\omega_{1}$ interacts with the system to produce a polarization at $3 \omega_{1}$. This is the process of third-harmonic generation. In general we deal with an incident field which is a superposition of four monochromatic waves whose frequencies are all different and which satisfy the relation $\omega_{1}+\omega_{2}+\omega_{3}=\omega_{4}$. For simplicity the electric dipole approximation is made again; $\exp ( \pm i \mathbf{k} \cdot \mathbf{r})=1$. The quantum-mechanical expression for the Fourier component of $\mathfrak{B}^{\mathrm{NL}}$ at $\omega_{4}$ which is proportional to $E_{1} E_{2} E_{3}$ is the following. For the sake of brevity we introduce the notation

$\frac{1}{\left(\omega_{k g} \mp \omega_{1}\right)\left(\omega_{j^{\prime} g} \pm \omega_{2} \pm \omega_{3}\right)\left(\omega_{j g} \pm \omega_{3}\right)}=\frac{1}{\left(\omega_{k g}-\omega_{1}\right)\left(\omega_{j^{\prime} g}+\omega_{2}+\omega_{3}\right)\left(\omega_{j g}+\omega_{3}\right)}+\frac{1}{\left(\omega_{k g}+\omega_{1}\right)\left(\omega_{j^{\prime} g}-\omega_{2}-\omega_{3}\right)\left(\omega_{j g}-\omega_{3}\right)}$,

etc.

$$
\begin{aligned}
& \mathfrak{P}_{l^{\mathrm{NL}}}\left(\omega_{4}\right)=\frac{\cos \left(\omega_{4} t+\phi_{1}+\phi_{2}+\phi_{3}\right)}{4 \hbar^{3}} \times \sum_{k, j^{\prime} j}\left\{\frac{\mathfrak{P}_{l g k}\left(\mathfrak{P}_{k j^{\prime}} \cdot \mathbf{E}_{1}\right)\left(\mathfrak{P}_{j^{\prime} j} \cdot \mathbf{E}_{2}\right)\left(\mathfrak{B}_{j g} \cdot \mathbf{E}_{3}\right)}{\left(\omega_{k g} \pm \omega_{4}\right)\left(\omega_{j^{\prime} g} \pm \omega_{2} \pm \omega_{3}\right)\left(\omega_{j g} \pm \omega_{3}\right)}+\frac{\mathfrak{P}_{l g k}\left(\mathfrak{P}_{k j^{\prime}} \cdot \mathbf{E}_{2}\right)\left(\mathfrak{P}_{j^{\prime} j} \cdot \mathbf{E}_{1}\right)\left(\mathfrak{P}_{j g} \cdot \mathbf{E}_{3}\right)}{\left(\omega_{k g} \pm \omega_{4}\right)\left(\omega_{j^{\prime} g} \pm \omega_{1} \pm \omega_{3}\right)\left(\omega_{j g} \pm \omega_{3}\right)}\right. \\
& +\frac{\mathfrak{P}_{l g k}\left(\mathfrak{P}_{k j^{\prime}} \cdot \mathbf{E}_{3}\right)\left(\mathfrak{P}_{j^{\prime} j} \cdot \mathbf{E}_{1}\right)\left(\mathfrak{P}_{j g} \cdot \mathbf{E}_{2}\right)}{\left(\omega_{k g} \pm \omega_{4}\right)\left(\omega_{j^{\prime} g} \pm \omega_{1} \pm \omega_{2}\right)\left(\omega_{j g} \pm \omega_{2}\right)}+\frac{\mathfrak{P}_{l g k}\left(\mathfrak{P}_{k j^{\prime}} \cdot \mathbf{E}_{1}\right)\left(\mathfrak{P}_{j^{\prime} j^{\prime}} \cdot \mathbf{E}_{3}\right)\left(\mathfrak{P}_{j g} \cdot \mathbf{E}_{2}\right)}{\left(\omega_{k g} \pm \omega_{4}\right)\left(\omega_{j^{\prime} g} \pm \omega_{2} \pm \omega_{3}\right)\left(\omega_{j g} \pm \omega_{2}\right)} \\
& +\frac{\mathfrak{P}_{l g k}\left(\mathfrak{P}_{k j^{\prime}} \cdot \mathbf{E}_{2}\right)\left(\mathfrak{P}_{j^{\prime} j} \cdot \mathbf{E}_{3}\right)\left(\mathfrak{P}_{j g} \cdot \mathbf{E}_{1}\right)}{\left(\omega_{k g} \pm \omega_{4}\right)\left(\omega_{j^{\prime} g} \pm \omega_{1} \pm \omega_{3}\right)\left(\omega_{j g} \pm \omega_{1}\right)}+\frac{\mathfrak{P}_{l g k}\left(\mathfrak{P}_{k j^{\prime}} \cdot \mathbf{E}_{3}\right)\left(\mathfrak{P}_{j^{\prime} j} \cdot \mathbf{E}_{2}\right)\left(\mathfrak{P}_{j g} \cdot \mathbf{E}_{1}\right)}{\left(\omega_{k g} \pm \omega_{4}\right)\left(\omega_{j^{\prime} g} \pm \omega_{1} \pm \omega_{2}\right)\left(\omega_{j g} \pm \omega_{1}\right)} \\
& +\frac{\mathfrak{P}_{l k j^{\prime}}\left(\mathfrak{P}_{k g} \cdot \mathbf{E}_{1}\right)\left(\mathfrak{P}_{j^{\prime} j} \cdot \mathbf{E}_{2}\right)\left(\mathfrak{P}_{j g} \cdot \mathbf{E}_{3}\right)}{\left(\omega_{k g} \mp \omega_{1}\right)\left(\omega_{j^{\prime} g} \pm \omega_{2} \pm \omega_{3}\right)\left(\omega_{j g} \pm \omega_{3}\right)}+\frac{\mathfrak{P}_{l k j^{\prime}}\left(\mathfrak{P}_{k g} \cdot \mathbf{E}_{2}\right)\left(\mathfrak{P}_{j^{\prime} j} \cdot \mathbf{E}_{1}\right)\left(\mathfrak{P}_{j g} \cdot \mathbf{E}_{3}\right)}{\left(\omega_{k g} \mp \omega_{2}\right)\left(\omega_{j^{\prime} g} \pm \omega_{1} \pm \omega_{3}\right)\left(\omega_{j g} \pm \omega_{3}\right)} \\
& +\frac{\mathfrak{P}_{l k j^{\prime}}\left(\mathfrak{P}_{k g} \cdot \mathbf{E}_{3}\right)\left(\mathfrak{P}_{j^{\prime} j} \cdot \mathbf{E}_{1}\right)\left(\mathfrak{P}_{j g} \cdot \mathbf{E}_{2}\right)}{\left(\omega_{k g} \mp \omega_{3}\right)\left(\omega_{j^{\prime} g} \pm \omega_{1} \pm \omega_{2}\right)\left(\omega_{j g} \pm \omega_{2}\right)}+\frac{\mathfrak{P}_{l k j^{\prime}}\left(\mathfrak{P}_{k g} \cdot \mathbf{E}_{1}\right)\left(\mathfrak{P}_{j^{\prime} j} \cdot \mathbf{E}_{3}\right)\left(\mathfrak{P}_{j g} \cdot \mathbf{E}_{2}\right)}{\left(\omega_{k g} \mp \omega_{1}\right)\left(\omega_{j^{\prime} g} \pm \omega_{2} \pm \omega_{3}\right)\left(\omega_{j g} \pm \omega_{2}\right)} \\
& \left.+\frac{\mathfrak{P}_{l k j^{\prime}}\left(\mathfrak{P}_{k g} \cdot \mathbf{E}_{2}\right)\left(\mathfrak{P}_{j^{\prime} j} \cdot \mathbf{E}_{3}\right)\left(\mathfrak{P}_{j g} \cdot \mathbf{P}_{1}\right)}{\left(\omega_{k g} \mp \omega_{2}\right)\left(\omega_{j^{\prime} g} \pm \omega_{1} \pm \omega_{3}\right)\left(\omega_{j g} \pm \omega_{1}\right)}+\frac{\mathfrak{P}_{l k j^{\prime}}\left(\mathfrak{P}_{k g} \cdot \mathbf{E}_{3}\right)\left(\mathfrak{P}_{j^{\prime} j} \cdot \mathbf{E}_{2}\right)\left(\mathfrak{P}_{j g} \cdot \mathbf{E}_{1}\right)}{\left(\omega_{k g} \mp \omega_{3}\right)\left(\omega_{j^{\prime} g} \pm \omega_{1} \pm \omega_{2}\right)\left(\omega_{j g} \pm \omega_{1}\right)}\right\}
\end{aligned}
$$

Equation (2.19) defines $\mathfrak{P}^{\mathrm{NL}}\left(\omega_{4}\right)$ as

$$
\mathfrak{P}_{l}^{\mathrm{NL}}\left(\omega_{4}\right)=\cos \left(\omega_{4} t+\phi_{1}+\phi_{2}+\phi_{3}\right) \sum_{m, n, p} \gamma_{l m n p}\left(\omega_{4}=\omega_{1}+\omega_{2}+\omega_{3}\right) E_{1 m} E_{2 n} E_{3 p},
$$

where the frequencies are all different. The $\gamma_{l m n p}$ are not zero for the case where the system has an inversion center and the wave functions have well-defined parity. The tensor $\gamma_{l m n p}$ has $3 \times 27$ components for the case of $C_{1}$ symmetry. The $\gamma$ 's for the other three components of polarization (as a function of three different fields) are obtained from the $\gamma\left(\omega_{4}\right)$ by the same type of permutations used in the three-wave case.

$$
\gamma_{l m n p}(4=1+2+3)=\gamma_{m l n p}(1=4-2-3)=\gamma_{n l m p}(2=4-1-3)=\gamma_{p l m n}(3=4-1-2)
$$

The various special cases in which the four frequencies satisfy additional relations among themselves are most conveniently treated individually, rather than as special cases of Eq. (3.19). The most important of these is the case $\omega_{1}=\omega_{2}=\omega_{3}=\frac{1}{3} \omega_{4}$. We define a new incident field $E=E_{1} \cos \left(\omega_{1} t+\phi_{1}\right)+E_{2} \cos \left(3 \omega_{1} t+\phi_{2}\right)$ and calculate the term in $\mathfrak{B}^{\mathrm{NL}}\left(3 \omega_{1}\right)$ which is proportional to $E_{1}^{3}$ and the term in $\mathfrak{P}^{\mathrm{NL}}\left(\omega_{1}\right)$ which is proportional to $E_{1}{ }^{2} E_{2}$. The expressions are

$\mathfrak{P}_{l^{\mathrm{NL}}}\left(3 \omega_{1}\right)=\frac{\cos \left(3 \omega_{1} t+3 \phi_{1}\right)}{4 \hbar^{3}} \times \sum_{k, j^{\prime}, j}\left\{\frac{\mathfrak{P}_{l g k}\left(\mathfrak{P}_{k j^{\prime}} \cdot \mathbf{E}_{1}\right)\left(\mathfrak{P}_{j^{\prime} j} \cdot \mathbf{E}_{1}\right)\left(\mathfrak{P}_{j g} \cdot \mathbf{E}_{1}\right)}{\left(\omega_{k g} \pm 3 \omega_{1}\right)\left(\omega_{j^{\prime} g} \pm 2 \omega_{1}\right)\left(\omega_{j g} \pm \omega_{1}\right)}+\frac{\mathfrak{P}_{l k j^{\prime}}\left(\mathfrak{P}_{k g} \cdot \mathbf{E}_{1}\right)\left(\mathfrak{P}_{j^{\prime} j} \cdot \mathbf{E}_{1}\right)\left(\mathfrak{P}_{j g} \cdot \mathbf{E}_{1}\right)}{\left(\omega_{k g} \mp \omega_{1}\right)\left(\omega_{j^{\prime} g} \pm 2 \omega_{1}\right)\left(\omega_{j g} \pm \omega_{1}\right)}\right\}$ 


$$
\begin{aligned}
\mathfrak{P}^{\mathrm{NL}}\left(\omega_{1}\right)=\frac{\cos \left(\omega_{1} t+2 \phi_{1}-\phi_{2}\right)}{4 \hbar^{3}} \times \sum_{k, j, j}\left\{\frac{\mathfrak{P}_{l g k}\left(\mathfrak{P}_{k j^{\prime}} \cdot \mathbf{E}_{1}\right)\left(\mathfrak{P}_{j^{\prime} j} \cdot \mathbf{E}_{1}\right)\left(\mathfrak{P}_{j g} \cdot \mathbf{E}_{2}\right)}{\left(\omega_{k g} \pm \omega_{1}\right)\left(\omega_{j^{\prime}} \pm 2 \omega_{1}\right)\left(\omega_{j g} \pm 3 \omega_{1}\right)}+\frac{\mathfrak{P}_{l g k}\left(\mathfrak{P}_{k j^{\prime}} \cdot \mathbf{E}_{1}\right)\left(\mathfrak{P}_{j^{\prime} j} \cdot \mathbf{E}_{2}\right)\left(\mathfrak{P}_{j g} \cdot \mathbf{E}_{1}\right)}{\left(\omega_{k g} \pm \omega_{1}\right)\left(\omega_{j^{\prime} g} \pm 2 \omega_{1}\right)\left(\omega_{j g} \mp \omega_{1}\right)}\right. \\
+\frac{\mathfrak{P}_{l g k}\left(\mathfrak{P}_{k j^{\prime}} \cdot \mathbf{E}_{2}\right)\left(\mathfrak{P}_{j^{\prime} j} \cdot \mathbf{E}_{1}\right)\left(\mathfrak{P}_{j g} \cdot \mathbf{E}_{1}\right)}{\left(\omega_{k g} \mp \omega_{1}\right)\left(\omega_{j^{\prime} g} \pm 2 \omega_{1}\right)\left(\omega_{j g} \pm \omega_{1}\right)}+\frac{\mathfrak{P}_{l k j^{\prime}}\left(\mathfrak{P}_{k g} \cdot \mathbf{E}_{1}\right)\left(\mathfrak{P}_{j^{\prime} j^{\prime}} \cdot \mathbf{E}_{2}\right)\left(\mathfrak{P}_{j g} \cdot \mathbf{E}_{1}\right)}{\left(\omega_{k g} \pm \omega_{1}\right)\left(\omega_{j^{\prime} g} \pm 2 \omega_{1}\right)\left(\omega_{j g} \mp \omega_{1}\right)} \\
+\frac{\mathfrak{P}_{l k j^{\prime}}\left(\mathfrak{P}_{k g} \cdot \mathbf{E}_{1}\right)\left(\mathfrak{P}_{j^{\prime} j^{\prime}} \cdot \mathbf{E}_{1}\right)\left(\mathfrak{P}_{j g} \cdot \mathbf{E}_{2}\right)}{\left(\omega_{k g}-\omega_{1}\right)\left(\omega_{j^{\prime} g}-2 \omega_{1}\right)\left(\omega_{j g}-3 \omega_{1}\right)}+\frac{\mathfrak{P}_{l k j^{\prime}}\left(\mathfrak{P}_{k g} \cdot \mathbf{E}_{2}\right)\left(\mathfrak{P}_{j^{\prime} j} \cdot \mathbf{E}_{1}\right)\left(\mathfrak{P}_{j g} \cdot \mathbf{E}_{1}\right)}{\left(\omega_{k g}+3 \omega_{1}\right)\left(\omega_{j^{\prime} g}-2 \omega_{1}\right)\left(\omega_{j g}-\omega_{1}\right)} \\
\left.+\frac{\mathfrak{P}_{l k j^{\prime}}\left(\mathfrak{P}_{k g} \cdot \mathbf{E}_{1}\right)\left(\mathfrak{P}_{j^{\prime} j} \cdot \mathbf{E}_{1}\right)\left(\mathfrak{P}_{j g} \cdot \mathbf{E}_{2}\right)}{\left(\omega_{k g}+\omega_{1}\right)\left(\omega_{j^{\prime} g}+2 \omega_{1}\right)\left(\omega_{j g}+3 \omega_{1}\right)}+\frac{\mathfrak{P}_{l k j^{\prime}}\left(\mathfrak{P}_{k g} \cdot \mathbf{E}_{2}\right)\left(\mathfrak{P}_{j^{\prime} j} \cdot \mathbf{E}_{1}\right)\left(\mathfrak{P}_{j g} \cdot \mathbf{E}_{1}\right)}{\left(\omega_{k g}+3 \omega_{1}\right)\left(\omega_{j^{\prime} g}+2 \omega_{1}\right)\left(\omega_{j g}+\omega_{1}\right)}\right\} .
\end{aligned}
$$

\section{Nonlinear Ionic Source Terms}

The previous treatment has dealt only with $\mathfrak{P}^{N L}$ due to electronic motions within atoms or molecules. In general the electric polarization of a system has ionic contributions as well, due to the forced vibrations of charged ions. If the ions vibrate in an anharmonic potential, they will make a contribution to $\mathfrak{P}^{\mathrm{NL}}$.

For the case of second harmonic generation the nonlinear ionic polarization is calculated as follows. A simplified model of the vibrations of the system is assumed; a given molecule is treated as a collection of three independent one-dimensional anharmonic oscillators. In the absence of radiation the Hamiltonian of the system is:

$$
\begin{array}{r}
\mathcal{H C}_{0}+\mathcal{H C}^{\prime}=\frac{1}{2} \sum_{i=x, y, z}\left(\frac{p_{i}{ }^{2}}{m_{i}}+m_{i} \omega_{0 i}{ }^{2} r_{i}{ }^{2}\right)-\frac{1}{3} \sum_{i=x, y, z}\left(\lambda_{i} r_{i}{ }^{3}\right) \\
-\frac{1}{4} \sum_{i=x, y, z} \eta_{i} r_{i}^{4} .
\end{array}
$$

In the electric dipole approximation the interaction of the system with radiation is:

where

$$
\mathfrak{H}_{\text {int }}=-\sum_{i=x, y, z} e_{i} r_{i} E_{\imath}(t)
$$

$$
\mathbf{E}=\mathbf{E}_{1} \cos \left(\omega_{1} t+\phi_{1}\right)+E_{2} \cos \left(2 \omega_{1} t+\phi_{2}\right) .
$$

There are two procedures for calculating $\left\langle\mathfrak{P}_{i}{ }^{\mathrm{NL}}\right\rangle$. One may use fourth-order perturbation theory involving matrix elements of $\mathfrak{H C}^{\prime}$ once, and of $\mathfrak{H C}_{\text {int }}$ three times. Alternatively, one may first use $\mathcal{H C}^{\prime}$ to calculate the wave functions of the anharmonic oscillators to first order in the $\lambda_{2}{ }^{22}$ These perturbed wave functions are then used in Eq. (2.17). In either case the expressions for the part of $\mathfrak{B}_{x} \mathrm{NL}$ due to $E_{1 x} E_{1 x}$ is

$$
\begin{aligned}
\beta_{x x x^{\text {ionic }}}\left(\omega_{2}\right. & \left.=\omega_{1}+\omega_{1}\right) E_{1 x^{2}} \cos \left(2 \omega_{1} t+2 \phi_{1}\right) \\
& =\frac{1}{2}\left(\frac{e_{x}}{m_{x}}\right)^{3} \lambda_{x} \frac{E_{1 x^{2}} \cos \left(2 \omega_{1} t+2 \phi_{1}\right)}{\left(\omega_{0 x^{2}}-^{2} \omega_{1}^{2}\right)^{2}\left(\omega_{0 x}{ }^{2}-4 \omega_{1}^{2}\right)} .
\end{aligned}
$$

This expression was obtained quantum mechanically,

\footnotetext{
${ }^{22}$ E. Hutchisson, Phys. Rev. 37, 45 (1931).
}

but it is exactly the result which would be obtained for this part of $\mathfrak{P}_{x}{ }^{\mathrm{NL}}$ using a purely classical calculation.

This classical calculation is now described briefly and applied to the generation of third harmonics by an anharmonic oscillator. The explicit quantum-mechanical calculation has not been done, but the results are almost certainly identical with what follows. Consider the anharmonic oscillator in the $x$ direction described by the $x$ part of Eq. (2.24). Write the classical equations of motion of this oscillator in a forcing field $E_{1 x} \cos \left(\omega_{1} t+\phi_{1}\right)$. Assume the solution for $x$ can be written in the form

$x=x_{1} \cos \left(\omega_{1} t+\phi_{1}\right)+x_{2} \cos \left(2 \omega_{1} t+2 \phi_{1}\right)$

One finds easily that $+x_{3} \cos \left(3 \omega_{1} t+3 \phi_{1}\right)$.

$$
\begin{aligned}
& \mathfrak{P}_{x} \mathrm{NL}\left(3 \omega_{1}\right)=e_{x} x_{3}(t)=E_{1}^{3} \cos \left(3 \omega_{1} t+3 \phi_{1}\right)\left(\frac{e_{x}}{m}\right)^{4} \\
& \times {\left[\frac{\lambda_{x}^{2}}{2 m} \frac{1}{\left(\omega_{1}^{2}-\omega_{0 x}^{2}\right)^{3}\left(\omega_{0}^{2}-4 \omega_{1}^{2}\right)\left(\omega_{0}^{2}-9 \omega_{1}^{2}\right)}\right.} \\
&\left.+\frac{\eta_{x}}{4} \frac{1}{\left(\omega_{1}^{2}-\omega_{0 x}^{2}\right)^{3}\left(\omega_{0 x}^{2}-9 \omega_{1}^{2}\right)}\right]
\end{aligned}
$$

However, since for ordinary vibrations the ratio $\left(2 \lambda^{2} / m \eta\right)\left[1 /\left(\omega_{0 x}^{2}-4 \omega_{1}^{2}\right)\right]$ is of the order of $10^{-2}-10^{-3}$, if $\omega_{1}$ is an optical frequency, only the second term in Eq. (2.26) is of consequence in producing thirdharmonic polarization.

\section{PHYSICAL RELATIONSHIPS BETWEEN MACROSCOPIC FIELD OUANTITIES IN NONLINEAR DIELECTRICS}

\section{A. Gases}

When the optical index of refraction is close to unity, the distinction between the microscopic field acting on an individual molecule and the macroscopic field is negligible. This situation occurs truly only in the case of gases. The molecules are subjected to an electromagnetic field by Eqs. (2.1) and (2.3). The effective nonlinear polarization in a volume element centered 
around the point $\mathbf{r}_{0}$ of the gas is obtained by averaging the effective dipole moment of a molecule, given by Eqs. (2.7) to (2.10), over all occupied states $g$ with the appropriate statistical weight and multiplying by the number of molecules $N$ per unit volume. With the introduction of $\Delta \mathbf{k}=\mathbf{k}_{1}+\mathbf{k}_{2}-\mathbf{k}_{3}$ and $\Delta \phi=\phi_{1}+\phi_{2}-\phi_{3}$ one obtains for the effective nonlinear polarization at $\mathbf{r}_{0}$,

$$
\begin{aligned}
\mathbf{P}^{\mathrm{NL}}\left(\omega_{3}\right)=\frac{1}{2} \operatorname{Re} & {\left[N Z^{-1} \frac{i q_{1} q_{2} e^{3}}{\omega_{3} C^{2} m^{3} \hbar^{2}} e^{i\left(\Delta \mathrm{k} \cdot \mathrm{r}_{0}+\Delta \phi\right)} e^{i\left(\mathrm{k}_{3} \mathrm{r}_{0}-\omega_{3} t+\phi_{3}\right)}\right.} \\
& \left.\times \sum_{g}[\mathfrak{A}+\mathfrak{B}]_{g g} e^{-W / k T}\right],
\end{aligned}
$$

where $Z$ is the partition function and $[\mathfrak{A}+\mathfrak{B}]_{g g}$ is given by Eqs. (2.9) and (2.10).

The nonlinear polarization at $\omega_{3}$ will, in general, have a component parallel to and in phase with the electric field

$$
\mathbf{E}_{3}=\operatorname{Re}\left\{\frac{i \omega_{3} q_{3} \hat{a}_{3}}{c} e^{i\left(\mathrm{k}_{3} \cdot \mathrm{r}_{0}-\omega_{3} t+\phi_{3}\right)}\right\}
$$

and a component parallel to, but $90^{\circ}$ out of phase with, $\mathbf{E}_{3}$.

The in-phase component will alter the effective dielectric constant of the wave at $\omega_{3}$ by an amount $\Delta \epsilon_{3}{ }^{\mathrm{NL}}=P^{\mathrm{NL}}\left(\omega_{3}\right.$, in phase) $q_{3}{ }^{-1} \omega_{3}{ }^{-1} c$. The out-of-phase component represents a gain or loss of intensity of the wave at $\omega_{3}$. This fact is of foremost practical importance. It accounts for the generation of light harmonics, mixing, etc.

The Eqs. (2.9) and (2.10) contain the influence of all multipole moments of the molecule. Since a gas is an isotropic medium, the average nonlinear source term will vanish if only electric dipole terms are retained, i.e., if $\exp (i \mathbf{k} \cdot \mathbf{r})$ is put equal to unity in all matrix elements. Note that $[\mathfrak{A}+\mathfrak{B}]$ is real for pure electric dipole terms. If, however, one of the three matrix elements has electric quadrupole (or magnetic dipole) character and the other two retain the electric dipole character, a fourthrank tensor relationship exists between $\mathbf{P}_{\text {eff }}{ }^{\mathrm{NL}}$ on the left and three vectors on the right, $\hat{a}_{1}, \hat{a}_{2}$, and a linear combination of $\mathbf{k}_{1}, \mathbf{k}_{2}$, and $\mathbf{k}_{3}$. In this case $[\mathfrak{A}+\mathfrak{B}]$ is pure imaginary.

The components $\mathbf{P}_{\text {eff }}{ }^{\mathrm{NL}}\left(\omega_{3}\right)$ at right angles to $\hat{a}_{3}$ will create (or attenuate) either waves at $\omega_{3}$ with the same $\mathbf{k}_{3}$, but another polarization, or waves at $\omega_{3}$ with a different direction of $\mathbf{k}_{3}$. Furthermore, polarization components at the difference frequency $\omega_{2}-\omega_{1}$ will create waves at that frequency with different polarizations; the new waves interfere with the old ones to create still more waves, etc. It would seem hardly justifiable to restrict the problem to just three or four waves. This can, nevertheless, be done if there is only one set of waves for which $\Delta \mathbf{k}=0$, or at least very much smaller than for other possible sets. Although initially several other waves may be created, only the one with $\Delta \mathbf{k}=\mathbf{0}$ will continue to grow. The phase factor $\exp \left(-i \Delta \mathbf{k} \cdot \mathbf{r}_{0}\right)$ for the other waves will change sign after they have traveled a certain distance $\Delta \mathbf{r}$.

At that point, the term $P^{\mathrm{NL}}$ (out-of-phase) in Eq. (2.8) changes sign, and the generation is followed by attenuation. This process will be analyzed in detail in the following sections.

Two examples to illustrate these remarks will describe how such situations may arise even in a gaseous medium. It will be assumed that only electric dipole matrix elements are of importance. In that case, it is necessary to go to fourth order in the electric field amplitudes $q \omega / c$.

Consider first the case that a strong dc electric field is applied to the gas. One may use the fourth-order expression for $P_{\text {eff }} \mathrm{NL}^{\mathrm{L}}$ in Eq. (2.19). Now however, one of the frequencies, say $\omega_{1}$, is zero. One redefines the frequencies and fields as follows: $E_{1} \rightarrow E_{\mathrm{dc}}, \omega_{2} \rightarrow \omega_{1}{ }^{\prime}$, $\omega_{3} \rightarrow \omega_{2}^{\prime}$, and $\omega_{4} \rightarrow \omega_{3}^{\prime}=\omega_{1}^{\prime}+\omega_{2}^{\prime}$.

Assume that all these fields are polarized in the $x$ direction. Because of the symmetry properties of the fourth-order tensor in an isotropic medium (averaging over all orientations of the molecules) Eq. (2.19) leads to an induced polarization in the $x$ direction. With the three waves all propagating in the $z$ direction, the nonlinear polarization becomes

$$
\begin{aligned}
\mathbf{P N L}^{\mathrm{N}}\left(\omega_{3}\right)= & N Z^{-1} \sum_{g}[]_{g g} e^{-W_{g} / k T} E_{0} E_{1} E_{2} \\
\times & {\left[\cos \left(k_{3} z-\omega_{3} t\right) \cos (\Delta k z+\Delta \phi)\right.} \\
& \left.-\sin \left(k_{3} z-w_{3} t\right) \sin (\Delta k z+\Delta \phi)\right],
\end{aligned}
$$

where the quantity []$_{g g}$ is real and is given by

$$
\frac{1}{4 \hbar^{3}} \sum_{k, j^{\prime}, j} P_{x g k} P_{x k j^{\prime}} P_{x j^{\prime} j} P_{x j g}\{\quad\}_{k j^{\prime} j},
$$

where \{\}$_{k j^{\prime} j}$ is given by Eq. (2.19), in which all numerators have been replaced by unity and the frequencies have been redefined as above. The frequencies $\omega_{1}$ and $\omega_{2}$ may be so chosen with respect to the known energy levels of the molecular species that the linear optical dispersion matches the phase velocities,

$$
|\Delta k|=\left|k_{3}-k_{1}-k_{2}\right| \ll\left|k_{1}\right| \text {. }
$$

The phase velocities are not matched for other combinations such as $\omega_{1}-\omega_{2}, \omega_{1}, \omega_{2}$, or $\omega_{3}+\omega_{1}, \omega_{3}, \omega_{1}$, etc. In this case, the problem is essentially restricted to the three linearly polarized waves mentioned above. One may, for example, take $\omega_{1}$ and $\omega_{2}$ to be frequencies just below the vibrational absorption band of a molecule such as $\mathrm{HCl}$. The summation in Eq. (3.3) then has to be carried over the states of an anharmonic oscillator. The dispersion associated with the vibrational absorption may lead to the condition of phase matching $k_{1}+k_{2}=k_{3}$. Experimentally, the matching can be facilitated by changing the pressure and admixture of other molecular species. Although the magnitude of the nonlinear polarization in a gas is clearly very much smaller than in a condensed medium, a similar reduction applies to the linear polarization. One can keep the phase velocities 
matched over a much longer distance in a gas. It may be argued that the ratio of nonlinear to linear polarization can be made more favorable in a gas than in a dense medium, because in the latter there will usually be many atoms or ions which do not contribute much to the nonlinearity, but are responsible for an appreciable fraction of the linear index of refraction.

Consider a point $z$ and initial phases of the waves $\phi_{1}, \phi_{2}$, and $\phi_{3}$, such that $\Delta k z+\Delta \phi=0$. The nonlinear polarization is then in phase with the electric field. There will be a nonlinear contribution to the dielectric constant. Keep the amplitudes $\mathbf{E}_{1}$ and $\mathbf{E}_{2}$ of the two light waves at $\omega_{1}$ and $\omega_{2}$ fixed. This gives a fixed amplitude $\mathbf{P N L}^{\mathrm{N}}\left(\omega_{3}\right)$ with a time factor in phase with the wave at $\omega_{3}$ with amplitude $\mathbf{E}_{3}$. If the amplitude of this wave is changed from 0 to $\mathbf{E}_{3}$, the time-averaged stored energy is changed by an amount $\frac{1}{2} \mathbf{P N L}^{\mathrm{NL}}\left(\omega_{3}\right) \cdot \mathbf{E}_{3}$ due to the nonlinearity of the medium. The factor $1 / 2$ comes from the time average of $\cos ^{2} \omega_{3} t$. The nonlinearity, therefore, causes a change in the dielectric constant of the wave at $\omega_{3}$ if the waves at $\omega_{1}$ and $\omega_{2}$ are present.

If $\Delta k z+\Delta \phi=\pi / 2$, there is no nonlinear contribution to the dielectric constant. In this case, the nonlinear polarization is exactly $90^{\circ}$ out of phase with the wave at $\omega_{3}$. It does positive or negative work on the wave. The work done per unit time by the nonlinear polarization of the material on the wave may be written as

$$
\begin{aligned}
W_{3}=\frac{\omega_{3}}{2 \pi} \int_{\text {eycle }} \mathbf{E}_{3} \frac{d \mathbf{P}^{N L}\left(\omega_{3}\right)}{d t} d t \\
=\frac{1}{2} \omega_{3} \mathbf{E}_{3} \mathbf{P N L}^{N}\left(\omega_{3}, \text { out-of-phase }\right) .
\end{aligned}
$$

As a consequence, the amplitude of the wave $\mathbf{E}_{3}$ will change.

If one starts out with two waves $\omega_{1}$ and $\omega_{2}$ and complete matching of the phase velocities, $\Delta k=0$, a wave at $\omega_{3}$ will start to grow with a phase $\phi_{3}=\phi_{2}+\phi_{1}+\frac{1}{2} \pi$. This phase will persist and the in-phase part of the nonlinear polarization will remain zero. The growth of the amplitude for this special case, as well as the general case of arbitrary initial amplitudes and phases and nonvanishing $\Delta k$ will be calculated in Secs. V-VII.

The other example in a gas, to which the same formalism can readily be applied, is the creation of the third harmonic wave. Consider a linearly polarized wave $E_{x} \cos \left(k_{1} z-\omega_{1} t\right)$. A polarization at the third harmonic $\omega_{3}=3 \omega$ will be created which is given in Eq. (2.26).

The even harmonics are not generated in the absence of a dc field. Other polarizations are likewise absent. The fifth harmonic will be assumed to have a poor phase match. The problem is thus restricted to two interacting electromagnetic waves. Detailed solutions for the amplitudes and phases will be given in Sec. VII. Energy relationships similar to the ones discussed above are readily derived. The free energy of a unit volume of gas simultaneously subjected to the fundamental and the third harmonic linearly polarized in the same direction has a term proportional to $E_{1}^{3} E_{3}$.
Phase matching can, in principle, be achieved by utilizing the resonance dispersion near absorption bands of the molecules. The anharmonic oscillator will lead to a very large resonant denominator if the fundamental is chosen just below the absorption band. The nonlinearity will then be proportional to $\left(\omega_{\text {vibr }}{ }^{2}-\omega_{1}^{2}\right)^{-3}$ according to Eq. (2.26).

\section{B. Isotropic and Cubic Dense Media}

In a dense medium the field acting on an atom or ion $j$ is the local field. Lorentz has shown that in a fluid, or in a position of cubic symmetry, one has

$$
\mathbf{E}_{\text {loc }}=\mathbf{E}+\frac{4}{3} \pi \mathbf{P}_{\text {tot }}=\mathbf{E}+\frac{4}{3} \pi \mathbf{P}^{\mathrm{L}}+\frac{4}{3} \pi \mathbf{P N}^{\mathrm{L}} \text {. }
$$

The macroscopic displacement vector $\mathbf{D}$, occurring in Maxwell's equations, is

$$
\mathbf{D}=\mathbf{E}+4 \pi \mathbf{P}^{\mathrm{L}}+4 \pi \mathbf{P}^{\mathrm{NL}} \text {. }
$$

The linear polarization can be expressed with the aid of the linear polarizability $\alpha$

$$
\mathbf{P}^{\mathrm{L}}=N \alpha \mathbf{E}_{\mathrm{loc}} .
$$

From Eqs. (3.5) and (3.7) it follows that

$$
\begin{aligned}
\mathbf{P}^{\mathrm{L}}=\frac{N \alpha}{1-\frac{4}{3} \pi N \alpha} & \mathbf{E}+\frac{N \alpha}{1-\frac{4}{3} \pi N \alpha} \frac{4 \pi}{3} \mathbf{P N L}^{\mathrm{L}} \\
& =[(\epsilon-1) / 4 \pi] \mathbf{E}+[(\epsilon-1) / 3] \mathbf{P}^{\mathrm{NL}} .
\end{aligned}
$$

Substitution into Eq. (3.6) yields

$$
\mathbf{D}=\epsilon \mathbf{E}+[(\epsilon+2) / 3] 4 \pi \mathbf{P}^{\mathrm{NL}}=\epsilon \mathbf{E}+4 \pi \mathbf{P}^{\mathrm{NLS}} .
$$

This equation shows the important result that the effective nonlinear polarization source term is $\frac{1}{3}(\epsilon+2)$ times the true nonlinear polarization which is calculated from

$$
\begin{aligned}
\mathbf{P}_{3}{ }^{N L}=N \beta \mathbf{E}_{\mathrm{loc}} \mathbf{E}_{\mathrm{loc}} & \\
& =N \beta\left[\left(\epsilon_{1}+2\right) / 3\right]\left[\left(\epsilon_{2}+2\right) / 3\right] \mathbf{E}_{1} \mathbf{E}_{2} .
\end{aligned}
$$

The difference has its origin in the dipolar energy between the nonlinear dipole moment at one lattice site and the linear dipole moment at another site. The presence of a nonlinear dipole moment at one site changes the local field and, therefore, the linear dipole moment at other sites. This in turn changes the dielectric displacement. The interaction between nonlinear dipole moments at different sites has been ignored in Eq. (3.9). This is consistent within the framework of this paper, in which the nonlinearity is considered as a small perturbation. It is smaller than the linear polarization by a factor $e E_{l} a /\left(W_{0}-W_{n}\right)$, as explained in the introduction. The combination of Eqs. (3.8) and (3.9) leads to a macroscopic nonlinear susceptibility

$$
\begin{aligned}
& x^{\mathrm{NL}}\left(\omega_{3}=\omega_{1}+\omega_{2}\right) \\
& =N \bigotimes\left(\omega_{3}=\omega_{1}+\omega_{2}\right) \frac{\epsilon\left(\omega_{1}\right)+2}{3} \frac{\epsilon\left(\omega_{2}\right)+2}{3} \frac{\epsilon\left(\omega_{3}\right)+2}{3} .
\end{aligned}
$$


This equation can easily be generalized to higher order relationships between four or more waves. Note that the macroscopic quantities $\chi$ follow the same symmetry relationship (Eq. 2.16) as the microscopic quantities $\beta$. The same arguments about energy stored in the dielectric and power transferred between the waves, given in the preceding section for gases, can now be repeated for the dense dielectric, provided $P^{\mathrm{NLS}}$ is taken as defined by Eq. (3.8). With this proviso, the discussion of the examples for gases go through without any modification for dense fluids and cubic crystals with centers of symmetry.

Cubic crystals with the $\mathrm{ZnS}$ structure which lack an inversion center will have a nonvanishing interaction between three waves, even if only electric dipole matrix elements are retained and no external dc electric field is applied. The third-order tensor $\chi_{i j k}$ has nonvanishing components, if all three indices are different, as in $\chi_{x y z}$. In such crystals, the second harmonic of light will readily be generated, but it will be difficult to correct the linear dispersion of the crystal in order to achieve a matching of the phase velocities of the fundamental and harmonic waves. Giordmaine ${ }^{13}$ and Maker, Terhune et al.,$^{14}$ have demonstrated how this matching may be achieved between ordinary and extraordinary rays in anisotropic crystals. The more complicated nonlinear relationships in these crystals will be taken up in the next paragraph. It should be kept in mind, however, that the basic physical ideas remain the same.

\section{Anisotropic Crystals}

The general case of an arbitrary number of atoms in the unit cell in a crystal of arbitrary symmetry can be attacked along the same lines. Instead of one scalar polarizability and one scalar Lorentz factor equal to $4 \pi / 3$, one now has many different tensors, which are discussed in the Appendix. Equations (A11) and (A13) of the Appendix are reproduced here for future use.

$$
\begin{gathered}
x\left(\omega_{3}=\omega_{2}+\omega_{1}\right) \\
=\sum_{i} \boldsymbol{\beta}^{(i)}\left(\omega_{3}=\omega_{2}+\omega_{1}\right) \\
\mathbf{P N}^{\mathrm{NLS}}\left(\omega_{3}\right)=x\left(\omega_{3}=\omega_{1}+\omega_{2}\right): \mathbf{E}_{1} \mathbf{E}_{2} .
\end{gathered}
$$

The macroscopic nonlinear susceptibility (Eq. 3.11) clearly satisfies the same permutation symmetry relationships (Eq. 2.16) possessed by the individual microscopic nonlinear polarizabilities $\boldsymbol{\beta}^{i}$. The secondorder tensors $\mathbf{N}$ are defined in the Appendix.

In addition, the macroscopic third-order nonlinear susceptibility tensor has the point symmetry properties of the crystal lattice as a whole, whereas the individual nonlinear polarizabilities have the symmetry properties of individual lattice sites. The procedure can again be generalized to higher order tensors.

The permutation symmetry relations allow the definition of a nonlinear part of the time average free energy density of the dielectric. This quantity can be defined, whereas the instantaneous value of the free energy has no meaning since in a dispersive medium the total polarization is not uniquely determined by the total field at each instant.

$$
\begin{aligned}
F & =U-T S-E P, \\
d F & =-S d T-P d E .
\end{aligned}
$$

The nonlinear part proportional to $E_{1} E_{2} E_{3}$ is given by a line integral in nine-dimensional space from the origin to the point $\mathbf{E}_{1}, \mathbf{E}_{2}, \mathbf{E}_{3}$

$$
\begin{aligned}
F^{\mathrm{NL}}=-\frac{1}{2} \int_{0}^{\mathbf{E}_{1,} \mathbf{E}_{2,} \mathbf{E}_{3}} d \mathbf{E}_{3} \cdot \chi\left(\omega_{3}=\omega_{1}+\omega_{2}\right): \mathbf{E}_{1} \mathbf{E}_{2} \\
+d \mathbf{E}_{2} \cdot \chi\left(\omega_{2}=\omega_{3}-\omega_{1}\right): \mathbf{E}_{3} \mathbf{E}_{1} \\
+d \mathbf{E}_{1} \cdot \varkappa\left(\omega_{1}=\omega_{3}-\omega_{2}\right): \mathbf{E}_{3} \mathbf{E}_{2} .
\end{aligned}
$$

Due to the permutation symmetry relations, this expression is independent of the path of integration. ${ }^{23}$ It does not matter in which order the three waves are switched on.

$$
\begin{aligned}
F^{\mathrm{NL}}=-\frac{1}{2} \mathbf{E}_{3} \cdot \mathbf{P}^{\mathrm{NLS}}\left(\omega_{3}\right)=-\frac{1}{2} \mathbf{E}_{2} \cdot \mathbf{P}^{\mathrm{NLS}}\left(\omega_{2}\right) \\
=-\frac{1}{2} \mathbf{E}_{1} \cdot \mathbf{P}^{\mathrm{NLS}}\left(\omega_{1}\right) .
\end{aligned}
$$

The factor $1 / 2$ comes from the time average. All quantities represent amplitudes. The nonlinear polarization at the three frequencies may be obtained by partial differentiation of $F^{\mathrm{NL}}$ with respect to the components of $\mathbf{E}_{1}, \mathbf{E}_{2}$, and $\mathbf{E}_{3}$, respectively. For the special case that one has only a linearly polarized fundamental wave $E_{1} \cos \omega_{1} t$ and a linearly polarized second harmonic $E_{2} \cos 2 \omega_{1} t$, one may write

$$
F^{\mathrm{NL}}=-\frac{1}{2} \chi E_{1}^{2} E_{2} .
$$

The nonlinear polarization has amplitudes given by

$$
\begin{aligned}
& P^{\mathrm{NLS}}\left(\omega_{1}\right)=2 \chi E_{1} E_{2}, \\
& P^{\mathrm{NLS}}\left(\omega_{2}\right)=\chi E_{1}{ }^{2} .
\end{aligned}
$$

A factor two has been restored to obtain amplitudes from the time-averaged free energy. These relationships can readily be generalized to quartic and higher order terms in $E$.

The energy density of the field caused by the introduction of the nonlinearity of the medium is

$$
\begin{aligned}
U_{\mathrm{field}}^{\mathrm{NL}}=\frac{1}{2} \int \mathbf{E} \cdot d \mathbf{P}^{\mathrm{NLS}} \text { (in phase) } \\
=\frac{1}{2} \sum_{i=1,2,3} \mathbf{E}_{i} \cdot \mathbf{P}_{i}^{\mathrm{NLS}} \text { (in phase) } \\
\quad-\frac{1}{2} \int \sum_{i} \mathbf{P}_{i}^{\mathrm{NLS}} \cdot d \mathbf{E}_{i} \\
=-3 F^{\mathrm{NL}}+F^{\mathrm{NL}}=-2 F^{\mathrm{NL}}
\end{aligned}
$$

${ }^{23}$ D. Widder, Advanced Calculus (Prentice-Hall Inc., Englewood Cliffs, New Jersey, 1947), p. 213. 
The same relationship holds for a fundamental wave and the second harmonic. The time-averaged field energy is minus two times the material free energy, if terms cubic in the field amplitudes are considered. For quartic terms, one has clearly

$$
U^{\mathrm{NL}}\left(E_{1}, E_{2}, E_{3}, E_{4}\right)=-3 F^{\mathrm{NL}}\left(E_{1}, E_{2}, E_{3}, E_{4}\right) .
$$

The out-of-phase components of the nonlinear polarization do not contribute to the free energy, but they are responsible for work done, as shown by Eq. (3.4). It should be noted that the $90^{\circ}$ out-of-phase components, with respect to the $E$ field at the same frequency, of $P^{N L S}$ at $\omega_{1}$ and $\omega_{2}$ have opposite signs from $P^{\mathrm{NLS}}\left(\omega_{3}\right.$, out-of-phase). The net work done by the nondissipative dielectric on the electromagnetic waves is zero. So is the total work done by the waves on the medium.

$$
\sum_{i} \omega_{i} \int_{\text {cycle }} \mathbf{E}_{i} \cdot \frac{d \mathbf{P}_{i} d t}{d t}=-\sum_{i} \omega_{i} \int_{\text {cycle }} \mathbf{P}_{i} \cdot \frac{d \mathbf{E}_{i}}{d t} d t=0
$$

These considerations for the time-averaged free energy in the presence of three electromagnetic waves can be extended to magnetic dipole and electric quadrupole effects. A crystal without inversion symmetry may, for example, have a term in the time-averaged free energy of the form ${ }^{24}$

$$
\begin{aligned}
F^{\mathrm{NL}}=\mathbf{E}_{3} \cdot \chi_{E H H}\left(\omega_{3}=\omega_{1}+\omega_{2}\right): \mathbf{H}_{1} \mathbf{H}_{2} \\
+\mathbf{E}_{2} \cdot \chi_{E H H}\left(\omega_{2}=\omega_{3}-\omega_{1}\right): \mathbf{H}_{3} \mathbf{H}_{1} \\
\quad+\mathbf{E}_{1} \cdot \chi \chi_{E H H}\left(\omega_{1}=\omega_{3}-\omega_{2}\right): \mathbf{H}_{3} \mathbf{H}_{2}
\end{aligned}
$$

with permutation symmetry relations of the type,

$\chi_{E i H_{j H k}}\left(\omega_{3}=\omega_{1}+\omega_{2}\right)=\chi_{H j E i H k}\left(\omega_{1}=\omega_{3}-\omega_{2}\right)$, etc.

Terms linear in $H$ will not occur, unless the crystal is ferromagnetic or antiferromagnetic and not invariant under time reversal.

The assumption of nondegenerate, linearly polarized modes has been made throughout this paper. The case of degenerate modes or circularly polarized modes has not been considered. When circular polarized light passes through an isotropic material, a sense of time is built in and a term linear in $H$ becomes important. Specifically, nonlinear effects of Faraday rotation have been excluded from our discussion.

In anisotropic crystals with a center of symmetry, the nonvanishing magnetic-electric dipole terms of lowest order are quadratic in $E$ and $H$. This term would, in general, be small, but might become of importance for the production of second harmonics or the interaction between three waves in the presence of a large static magnetic field.

In the absence of externally applied dc electric and magnetic fields, crystals with a center of symmetry still

${ }^{24} \mathrm{~N}$. Bloembergen, Proceedings of the Conference on High Magnetic Fields (Massachusetts Institute of Technology Press, Cambridge, 1962). have a term which is cubic in the field amplitudes. It is of the mixed dipolar-quadrupolar type, corresponding to a contribution to the time-averaged free energy $F^{\mathrm{NL}}=\mathbf{k}_{3} \mathbf{E}_{3}: \mathbf{Q}\left(\omega_{3}=\omega_{1}+\omega_{2}\right): \mathbf{E}_{1} \mathbf{E}_{2}$

$$
\begin{aligned}
+\mathbf{k}_{2} \mathbf{E}_{2}: \mathbf{Q}\left(\omega_{2}=\omega_{3}-\omega_{1}\right): \mathbf{E}_{3} \mathbf{E}_{1} \\
+\mathbf{k}_{1} \mathbf{E}_{1}: \mathbf{Q}\left(\omega_{1}=\omega_{3}-\omega_{2}\right): \mathbf{E}_{3} \mathbf{E}_{2}
\end{aligned}
$$

\section{THE COUPLING BETWEEN ELECTRO- MAGNETIC WAVES}

Maxwell's equations in the lossless, nonlinear dielectric medium can be written for each frequency component, as

$$
\begin{aligned}
& \nabla \times \mathbf{E}=-(1 / c)(\partial \mathbf{B} / \partial t) \\
& \nabla \times \mathbf{H}=+\frac{1}{c} \frac{\partial \mathbf{D}}{\partial t}=+\frac{1}{c} \frac{\partial \boldsymbol{\varepsilon} \cdot \mathbf{E}}{\partial t}+\frac{4 \pi}{c} \frac{\partial \mathbf{P}^{N L S}}{\partial t} .
\end{aligned}
$$

The nonlinear source term has been defined in the preceding sections. The energy balance may be written as a balance between the net flux into a volume element $V$ bounded by a surface $\sigma$ and the change in stored energy of the linear medium plus the work done by the nonlinear polarization on the linear wave at frequency $\omega_{3}$,

$$
\begin{aligned}
& -\frac{c}{4 \pi} \iint \mathbf{E}_{3} \times \mathbf{H}_{3} \cdot \hat{n} d \sigma \\
& =\frac{1}{8 \pi} \frac{\partial}{\partial t} \iiint\left\{\mathbf{E}_{3} \cdot \boldsymbol{\varepsilon}\left(\omega_{3}\right) \cdot \mathbf{E}_{3}+\mathbf{H}_{3} \cdot \mathbf{u}\left(\omega_{3}\right) \cdot \mathbf{H}_{3}\right\} d V \\
& \quad+\iiint \mathbf{E}_{3} \cdot \frac{d \mathbf{P}^{\mathrm{NLS}}\left(\omega_{3}\right)}{d t} d V
\end{aligned}
$$

All quantities are real in this expression and similar ones that can be written down at the frequencies $\omega_{1}$ and $\omega_{2}$. If the equations at the three frequencies are averaged over an integral number of cycles and added, the algebraic sum of the last terms on the right vanishes according to Eq. (3.20). The usual energy balance in a lossless dielectric results. On the left, one has the total power flow through $\sigma$. It is of more interest to consider the Poynting vector and energy balance near each frequency separately.

Consider a wave

$$
\begin{aligned}
\mathbf{E}_{3}=\operatorname{Re}\left[\hat{a}_{3} A_{3}(z) e^{i\left(k 3 z-\omega_{3} t\right)}\right] \\
\quad=\hat{a}_{3} \rho_{3}(z) \cos \left[k_{3} z-\omega_{3} t+\phi_{3}(z)\right] .
\end{aligned}
$$

In a linear medium, the complex amplitude of a plane wave is constant. In a nonlinear medium this complex amplitude will change due to interaction with other waves at frequencies $\omega_{1}$ and $\omega_{2}$. The wave normal is taken in the $z$ direction, which is quite arbitrary with respect to the crystallographic axes. In anisotropic crystals, the polarization vector $\hat{a}_{3}$ may make an angle 


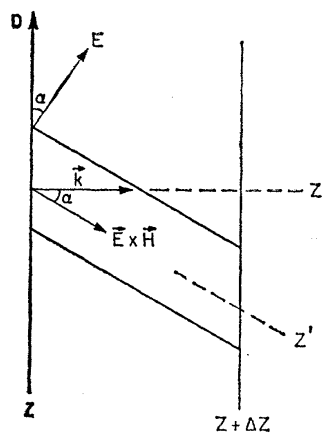

FIG. 1. The propagation of light in an anisotropic crystal. The phase normal is the $z$ direction. The energy flows in the ray direction $z^{\prime}$.

with the $z$ direction. The energy propagates along the direction $z^{\prime}$, in the plane defined by $\hat{a}_{3}$ and $\hat{z}$, normal to $\hat{a}_{3}$. The geometrical relationships between macroscopic field quantities are discussed in detail by Born and Wolf. ${ }^{25}$ In their notation, the angle between $z^{\prime}$ and $z$ is denoted by $\alpha$.

With $\mu=1$, Eq. (4.1) gives a magnetic field perpendicular to $\mathbf{E}$ and $\hat{z}$ with a magnitude $|H|=\rho_{3} k_{3} c \omega_{3}{ }^{-1} \cos \alpha$.

Consider the cylinder shown in Fig. 1 , bounded by planes at $z$ and $z+\Delta z$ with $1-\mathrm{cm}^{2}$ cross section. If Eq. (4.3) is integrated in time over an integral number of cycles, and spatially integrated over the cylinder, one obtains

$$
\begin{aligned}
& \frac{k_{3} c^{2}}{4 \pi \omega_{3}} \cos ^{2} \alpha \frac{d\left(\rho_{3}^{2}\right)}{d z} \Delta z \\
& \quad=\omega_{3} \rho_{3} \hat{a}_{3} \cdot \mathbf{P N L S}^{\mathrm{NLS}}\left(\omega_{3}, \text { out of phase }\right) \Delta z .
\end{aligned}
$$

Substituting Eq. (3.12) for $\mathbf{P}^{\mathrm{NLS}}$ leads to a differential equation for the amplitude of the wave at $\omega_{3}$,

$$
\frac{d \rho_{3}}{d z}=\frac{2 \pi \omega_{3}^{2}}{k_{3} c^{2}} \frac{\left(\hat{a}_{3} \cdot x^{\mathrm{NL}}: \hat{a}_{1} \hat{a}_{2}\right)}{\cos ^{2} \alpha_{3}} \rho_{1} \rho_{2} \sin (\Delta k z+\Delta \phi) .
$$

The notation for the phase difference between the three waves was introduced in Sec. III. The assumption has been made that the resultant of $\mathbf{k}_{1}+\mathbf{k}_{\mathbf{2}}$ is in the $z$ direction. In fact, in the remainder of this paper, the phase velocities of the three waves will be assumed to be all nearly parallel to each other except in the case of exact matching, $\mathbf{k}_{1}+\mathbf{k}_{\mathbf{2}}=\mathbf{k}_{\mathbf{3}}$, when the propagation vectors may have arbitrary directions. Effects arising from a small mismatch of the phase velocities along the $x$ and $y$ direction will be ignored.

The ray velocities will, of course, also be nearly parallel in this case. The physical interaction between light beams of finite cross section takes place along the direction of energy flow, $z^{\prime}$. This derivation shows the physical significance of the occurrence of the term $(\cos \alpha)^{-2}$. It also shows that the component of the nonlinear polarization parallel to the electric field vector is the true source. There is, of course, another

${ }^{25}$ M. Born and E. Wolf, Principles of Optics (Pergamon Press, New York, 1959), Chap. 14. wave, also propagating along $z$, with another linear polarization vector $\hat{a}_{3}{ }^{\prime}$. It will be assumed that the phase velocity for this wave is severely mismatched, $\Delta k^{\prime} \gg \Delta k$. This wave, as well as waves at other frequencies, are therefore ignored.

Equation (4.6) does not include the effect of the

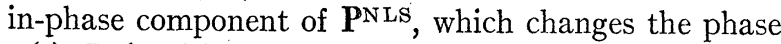
$\phi(z)$. It is of interest to derive the combined effect in a somewhat different manner, starting from the wave equation. A complex notation will be adopted for $\mathbf{E}_{3}$ and $\mathbf{P}_{3}{ }^{\text {NLS }}$, as defined by Eq. (4.4). This may safely be done after the nonlinear operation to obtain the real $\mathbf{P}^{\mathrm{NLS}}\left(\omega_{3}\right)$, with an in-phase and out-of-phase component, has been performed. The combination of Maxwell's Eqs. (4.1) and (4.2) leads to a wave equation with a nonlinear source term,

$$
\nabla \times \nabla \times \mathbf{E}+\frac{1}{c^{2}} \frac{\partial^{2}(\varepsilon \mathbf{E})}{\partial t^{2}}=-\frac{4 \pi}{c^{2}} \frac{\partial^{2} \mathbf{P}^{N L S}}{\partial t^{2}} .
$$

The left-hand side put equal to zero is the homogeneous equation, satisfied in a linear medium by the wave [Eq. (4.4)] with $A(z)$ taken constant, independent of $z$. The inhomogeneous equation can be satisfied if $A(z)$ obeys another set of differential equations, obtainable by straightforward substitution. In physically realizable situations, the relative change in the amplitude per wavelength is small, since the nonlinear susceptibility is very small compared to the linear part. Thus, terms in the second derivative of amplitude are negligible, $\partial^{2} A / \partial z^{2} \ll k \partial A / \partial z$. A scalar multiplication of Eq. (4.7) with $\hat{a}_{3}$ and substitution of Eq. (4.4) leads to a differential equation for the complex amplitude,

$$
\begin{aligned}
+2 \hat{a}_{3} \cdot\left(\nabla A_{3}\right) \times\left[\nabla \times \hat{a}_{3} \exp \left(i k_{3} z-\omega_{3} t\right)\right] \\
=\frac{4 \pi \omega_{3}^{2}}{c^{2}} \hat{a}_{3} \cdot x: \hat{a}_{1} \hat{a}_{2} A_{1} A_{2} \exp \left[i\left(k_{1}+k_{2}\right) z-i\left(\omega_{1}+\omega_{2}\right) t\right] .
\end{aligned}
$$

This equation can be transformed as follows,

$$
\begin{aligned}
& +2 \hat{a}_{3} \times\left[\nabla \times \hat{a}_{3} \exp \left(i k_{3} z-\omega_{3} t\right)\right] \cdot \nabla A_{3} \\
& =\left(4 \pi \omega_{3}^{2} / c^{2}\right)\left(\hat{a}_{3} \cdot x: \hat{a}_{1} \hat{a}_{2}\right) A_{1} A_{2} \exp \left[i\left(k_{1}+k_{2}\right) z-i \omega_{3} t\right] \\
& \quad+i k_{3} \cos ^{2} \alpha_{3}\left(\partial A_{3}(z) / \partial z^{\prime}\right) \\
& \quad=\left(2 \pi \omega_{3}^{2} / c^{2}\right)\left(\hat{a}_{3} \cdot x: \hat{a}_{1} \hat{a}_{2}\right) A_{1} A_{2} \exp i \Delta k z
\end{aligned}
$$

There is, of course, another equation of the same form and larger value of $\Delta k$ for the component of $\mathbf{E}$ in the direction of the other polarization vector. The third component of $\mathbf{E}$ can most conveniently be found from the condition

$$
\nabla \cdot \mathbf{D}=\nabla \cdot \mathbf{E}+4 \pi \nabla \cdot \mathbf{P N L S}^{\mathrm{N}}=0 .
$$

If the derivative in the direction of the wave normal and the real part of Eq. (4.8) is taken, the result is again Eq. (4.6). Similar differential equations may be derived for $A_{1}(z)$ and $A_{2}(z)$. Note that one has to combine $A_{3} \exp \left(i \omega_{3} t\right)$ with $A_{2}{ }^{*} \exp \left(-i \omega_{2} t\right)$ to get 
$A_{1} \exp \left(i \omega_{1} t\right)$, etc. The permutation symmetry relations make it possible to define a real constant

$K=\frac{2 \pi}{c^{2}} \hat{a}_{3} \cdot x\left(\omega_{3}\right): \hat{a}_{1} \hat{a}_{2}=\frac{2 \pi}{c^{2}} \hat{a}_{2} \cdot x\left(\omega_{2}\right): \hat{a}_{3} \hat{a}_{1}$

$=\frac{2 \pi}{c^{2}} \hat{a}_{1} \cdot \chi\left(\omega_{1}\right): \hat{a}_{3} \hat{a}_{2}$

and the coupled amplitude equations become

$$
\begin{aligned}
d A_{1}{ }^{*} / d z & =+i\left(\omega_{1}^{2} K / k_{1} \cos ^{2} \alpha_{1}\right) A_{3}{ }^{*} A_{2} e^{+i\left(\Delta_{k} z+\Delta \phi\right)} \\
d A_{2}{ }^{*} / d z & =+i\left(\omega_{2}^{2} K / k_{2} \cos ^{2} \alpha_{2}\right) A_{3}{ }^{*} A_{1} e^{+i\left(\Delta_{k} z+\Delta \phi\right)} \\
d A_{3} / d z & =-i\left(\omega_{3}^{2} K / k_{3} \cos ^{2} \alpha_{3}\right) A_{1} A_{2} e^{+i\left(\Delta_{k} z+\Delta \phi\right)}
\end{aligned}
$$

In a similar way one obtains the equations which describe the variations in amplitude and phase for a second harmonic interacting with the fundamental,

$$
\begin{aligned}
d A_{1}^{*} / d z & =+i\left(2 \omega^{2} K / k_{1} \cos ^{2} \alpha_{1}\right) A_{2}^{*} A_{1} e^{+i(2 k 1-k 2) z}, \\
d A_{2} / d z & =-i\left(4 \omega^{2} K / k_{2} \cos ^{2} \alpha_{2}\right) A_{1}^{2} e^{+i(2 k 1-k 2) z} .
\end{aligned}
$$

These equations can be generalized to higher powers in the field amplitudes. The amplitude equations for the third harmonic are, for example,

$$
\begin{array}{r}
d A_{1}{ }^{*} / d z=i\left(3 \omega^{2} / k_{1} \cos ^{2} \alpha_{1}\right)\left[C A_{3}{ }^{*} A_{1}^{2} e^{i(3 k 1-k 3) z}\right. \\
\left.+C^{\prime} A_{1} A_{1} A_{1}{ }^{*}+C^{\prime \prime} A_{1}{ }^{*} A_{3} A_{3}{ }^{*}\right] \\
d A_{3} / d z=-i\left(9 \omega^{2} / k_{3} \cos ^{2} \alpha_{3}\left[C A_{1}^{3} e^{+i\left(3 k_{1}-k 3\right) z}\right.\right. \\
\left.+3 C^{\prime \prime} A_{3} A_{1} A_{1}{ }^{*}+C^{\prime \prime \prime} A_{3} A_{3} A_{3}{ }^{*}\right] .
\end{array}
$$

The constant $C$ is given by

$$
\begin{aligned}
C=\left(2 \pi / c^{2}\right) \hat{a}_{3} \cdot x^{\prime}\left(\omega_{3}=\omega_{1}+\omega_{1}+\omega_{1}\right) \vdots \hat{a}_{1} \hat{a}_{1} \hat{a}_{1} \\
=\left(2 \pi / c^{2}\right) \hat{a}_{1} \cdot x^{\prime \prime}\left(\omega_{1}=\omega_{3}-\omega_{1}-\omega_{1}\right): \hat{a}_{3} \hat{a}_{1} \hat{a}_{1},
\end{aligned}
$$

with the fourth-rank tensor $\chi^{\prime}$ expressible in terms of the higher order nonlinear polarizability $\boldsymbol{\gamma}^{\prime}$ given by Eq. (2.22). The quantities $C^{\prime}, C^{\prime \prime}$, and $C^{\prime \prime \prime}$ can be referred in a similar way to the nonlinear polarizability. They occur in terms that are purely reactive in nature. It is clear from the notation that, e.g., the term $C^{\prime \prime} A_{1}{ }^{*} A_{3} A_{3}{ }^{*}=\partial A_{1}{ }^{*} / \partial z$ corresponds to a partial contribution to the coherent scattering by a quantum process, in which photons at $\omega_{1}$ and $\omega_{3}$ get scattered simultaneously. There is no change of the power flow involved. In fact, these reactive terms represent a quadratic dc Kerr effect. The propagation constant for the wave at $\omega_{3}$ changes in a similar way by a term proportional to $C^{\mathrm{dc}} E_{\mathrm{do}}{ }^{2}$ as by $C^{\prime \prime} A_{1} A_{1}{ }^{*}+C^{\prime \prime \prime} A_{3} A_{3}{ }^{*}$. This question will be discussed further in Sec. VII.

The third-harmonic situation is a special case of the interaction between four electromagnetic waves. If, for example, the frequency and momentum relationships

$$
\omega_{4}+\omega_{1}=\omega_{2}+\omega_{3}, \quad \Delta k=+k_{2}+k_{3}-k_{1}-k_{4}
$$

exist between the four waves, the coupled amplitude equations are

$$
\begin{gathered}
\frac{d A_{1}}{d z}=-i \frac{\omega_{1}^{2} C}{k_{1} \cos ^{2} \alpha_{1}} A_{2} A_{3} A_{4}{ }^{*} e^{i \Delta k z} \\
\quad-\frac{i \omega_{1}^{2}}{k_{1} \cos ^{2} \alpha_{1}} A_{1} \sum_{j=1}^{4} C_{1 j} A_{j} A_{j}{ }^{*}, \\
\frac{d A_{2}^{*}}{d z}=+i \frac{\omega_{2}{ }^{*} C}{k_{2} \cos ^{2} \alpha_{2}} A_{1}^{*} A_{3}{ }^{*} A_{4}{ }^{*} e^{i \Delta k z} \\
+\frac{i \omega_{2}^{2}}{k_{2} \cos ^{2} \alpha_{2}} A_{2}{ }^{*} \sum_{j=1}^{4} C_{2 j} A_{j} A_{j}{ }^{*},
\end{gathered}
$$

$$
\begin{gathered}
\frac{d A_{3}{ }^{*}}{d z}=+i \frac{\omega_{3}^{2} C}{k_{3} \cos ^{2} \alpha_{3}} A_{1}{ }^{*} A_{2} A_{4}{ }^{*} e^{i \Delta k z} \\
\quad+\frac{i \omega_{3}^{2}}{k_{3} \cos ^{2} \alpha_{3}} A_{3}{ }^{*} \sum_{j=1}^{4} C_{3 j} A_{j} A_{j}{ }^{*}, \\
\frac{d A_{4}}{d z}=-i \frac{\omega_{4}^{2} C}{k_{4} \cos ^{2} \alpha_{4}} A_{1} * A_{2} A_{3} e^{i \Delta k z} \\
-\frac{i \omega_{4}}{k_{4} \cos ^{2} \alpha_{4}} A_{4} \sum_{j=1}^{4} C_{4 j} A_{j} A_{j}{ }^{*} .
\end{gathered}
$$

The purely reactive, quadratic Kerr effect terms occur again on the right-hand side.

Some integrals of these complex amplitude equations can be obtained immediately by multiplying the equations by $A_{1}{ }^{*}, A_{2}, A_{3}$, and $A_{4}{ }^{*}$, respectively, and adding the complex conjugates. The right-hand sides become equal. Note that the component of the Poynting vector along the wave normal may be written as

$$
\begin{aligned}
\left|\mathbf{S}_{1}\right| \cos \alpha_{1}=\frac{c \cos \alpha_{1}}{8 \pi} \mid\left(\mathbf{E}_{1} \times \mathbf{H}_{1} *\right. & \left.+\mathbf{E}_{1} * \times \mathbf{H}_{1}\right) \mid \\
& =\frac{k_{1} c^{2} \cos ^{2} \alpha_{1}}{4 \pi \omega_{1}} A_{1} A_{1} *
\end{aligned}
$$

and similar relations at the other frequencies. In this manner, the Manley-Rowe relationships, ${ }^{26}$ well known in the theory of parametric amplifiers ${ }^{19}$ are obtained;

and

$$
\begin{aligned}
& \left|S_{1}\right| \cos \alpha_{1} / \omega_{1}+\left|S_{2}\right| \cos \alpha_{2} / \omega_{2}, \\
& \left|S_{1}\right| \cos \alpha_{1} / \omega_{1}+\left|S_{3}\right| \cos \alpha_{3} / \omega_{3},
\end{aligned}
$$

$$
\left|S_{1}\right| \cos \alpha_{1} / \omega_{1}-\left|S_{4}\right| \cos \alpha_{4} / \omega_{4}
$$

are "constants of the motion." The physical interpretation is that if the number of quanta passing through one $\mathrm{cm}^{2}$ of the wave front per second increases by a certain amount in the wave at $\omega_{1}$, the corresponding

${ }^{26} \mathrm{H}$. A. Haus, IRE Trans. on Microwave Theory and Tech. 6, 317 (1958). 
number of photons in the wave at $\omega_{4}$ increases by the same amount, and the corresponding number of photons at $\omega_{2}$ and $\omega_{3}$ decreases by this amount. ${ }^{27}$ The Manley-Rowe relations corresponding to Eqs. (4.9), (4.10), and (4.11) follow in the same manner. For a complete solution of the coupled nonlinear differential equations, it is necessary to return to the real notation. This will be carried out in the following sections.

\section{SECOND-HARMONIC GENERATION; EXACT SOLUTION}

Using the notation of Eq. (4.4) the complex Eq. (4.10) for second-harmonic generation can be written in their real and imaginary parts

$$
\begin{aligned}
d \rho_{1} / d z & =-\left(2 \omega^{2} K / k_{1} \cos ^{2} \alpha_{1}\right) \rho_{1} \rho_{2} \sin \theta, \\
d \rho_{2} / d z & =\left(4 \omega^{2} K / k_{2} \cos ^{2} \alpha_{2}\right) \rho_{1}^{2} \sin \theta, \\
d \theta / d z & =\Delta k-4 \omega^{2} K\left[\rho_{2} / k_{1} \cos ^{2} \alpha_{1}\right. \\
& \left.-\rho_{1}^{2} / \rho_{2} k_{2} \cos ^{2} \alpha_{2}\right] \cos \theta,
\end{aligned}
$$

where $\theta=2 \phi_{1}(z)-\phi_{2}(z)+\Delta k z$ and $\Delta k=2 k_{1}-k_{2}$. From the first two of these equations one obtains the ManleyRowe relation, which in the case of two waves is equivalent to the conservation of power flow in the lossless dielectric,

$$
W=\left(c^{2} / 8 \pi \omega\right)\left[k_{1} \rho_{1}^{2} \cos ^{2} \alpha_{1}+\frac{1}{2} k_{2} \rho_{2}^{2} \cos ^{2} \alpha_{2}\right] .
$$

Substitution of Eq. (5.1a) and Eq. (5.1b) into Eq. (5.1c) leads to

$$
d \theta / d z=\Delta k+(\cos \theta / \sin \theta)(d / d z) \ln \left(\rho_{1}^{2} \rho_{2}\right) .
$$

Further simplification can be obtained by the substitutions,

$$
\begin{aligned}
& u=\left[c^{2} k_{1} \cos ^{2} \alpha_{1} / 8 \pi \omega W\right]^{1 / 2} \rho_{1}, \\
& v=\left[c^{2} k_{2} \cos ^{2} \alpha_{2} / 16 \pi \omega W\right]^{1 / 2} \rho_{2}, \\
& \zeta=\left(2 \omega^{2} K / k_{1} \cos ^{2} \alpha_{1}\right)\left(16 \pi \omega W / c^{2} k_{2} \cos ^{2} \alpha_{2}\right)^{1 / 2} z .
\end{aligned}
$$

Equations (5.1) and (5.3) become

$$
\begin{aligned}
& d u / d \zeta=-u v \sin \theta \\
& d v / d \zeta=u^{2} \sin \theta \\
& d \theta / d \zeta=\Delta s+(\cos \theta / \sin \theta)(d / d \zeta) \ln \left(u^{2} v\right)
\end{aligned}
$$

where

$$
\Delta s=\frac{\Delta k}{\left(2 \omega^{2} K / k_{1} \cos ^{2} \alpha_{1}\right)\left(16 \pi \omega W / c^{2} k_{2} \cos ^{2} \alpha_{2}\right)^{1 / 2}},
$$

and Eq. (5.2) is now

$$
1=u^{2}+v^{2}
$$

\section{A. Matched Phase Velocities}

When the fundamental and second harmonic have equal phase velocities, i.e., $2 k_{1}=k_{2}$ and $\Delta k=\Delta s=0$, one can immediately integrate the third of Eqs. (5.5)

\footnotetext{
${ }^{27}$ M. T. Weiss, Proc. IRE (Inst. Radio Engrs.) 45, 1012 (1957).
}

to get a second constant of integration

$u^{2} v \cos \theta=\Gamma=\left(2 k_{1} / k_{2}\right)\left(c^{2} k_{2} / 16 \pi \omega W\right)^{2 / 2} \rho_{1}{ }^{2}(0) \rho_{2}(0)$

$$
\times \cos ^{2} \alpha_{1} \cos \alpha_{2} \cos \left[\phi_{2}(0)-2 \phi_{1}(0)\right] \text {. }
$$

From Eqs. (5.5), (5.7), and (5.8) one can obtain

$$
(d / d \zeta)\left(v^{2}\right)= \pm 2\left[v^{2}\left(1-v^{2}\right)^{2}-\Gamma^{2}\right]^{1 / 2}
$$

where the \pm sign is determined by the $\operatorname{sign}$ of $\sin \theta$ for $z=0$. The general solution to Eq. (5.9) can be written as the elliptic integral,

$$
\zeta= \pm \frac{1}{2} \int_{v^{2}(0)}^{v^{2}(\zeta)} \frac{d\left(v^{2}\right)}{\left[v^{2}\left(1-v^{2}\right)^{2}-\Gamma^{2}\right]^{1 / 2}}
$$

Since $v$ is real and less than or equal to $1, v^{2}$ is constrained to move between the two lowest positive roots of $v^{2}\left(1-v^{2}\right)^{2}-\Gamma^{2}=0$. Let us call these two roots $v_{a}$ and $v_{b}, v_{a} \leq v_{b}$; then $v$ oscillates between $v_{a}$ and $v_{b}$ with a period given by

$$
\Pi_{\zeta}=\int_{v_{a}^{2}}^{v_{b}^{2}} \frac{d\left(v^{2}\right)}{\left[v^{2}\left(1-v^{2}\right)^{2}-\Gamma^{2}\right]^{1 / 2}} .
$$

A typical solution is shown graphically in Fig. 2. The particular initial condition $\Gamma=0$ implies $v_{a}^{2}=0, v_{b}^{2}=1$, $\Pi_{\zeta} \rightarrow \infty$. In this case the integration of Eq. (5.10) is elementary

$$
\begin{gathered}
v_{\Gamma=0}(\zeta)=\tanh \left(\zeta+\zeta_{0}\right), \\
u_{\Gamma=0}(\zeta)=\operatorname{sech}\left(\zeta+\zeta_{0}\right) .
\end{gathered}
$$

The constant of integration $\zeta_{0}$ vanishes if the amplitude of the second harmonic is initially zero, $\rho_{2}(0)=0$. This is the often encountered initial condition, in which the second harmonic has to be generated. This solution is represented in Fig. 3. Correct treatment of the boundary conditions at the surface of the dielectric will lead, however, to a slightly different initial condition. In most crystals the values of $\cos ^{2} \alpha_{1}$, and $\cos ^{2} \alpha_{2}$ are close to unity. A characteristic interaction length $l$ can be defined by

$$
l^{-1} \approx 2 \omega^{2} K k_{1}^{-1} \rho_{1}(0) .
$$

In this distance about $75 \%$ of the fundamental power will have been converted to the second harmonic.

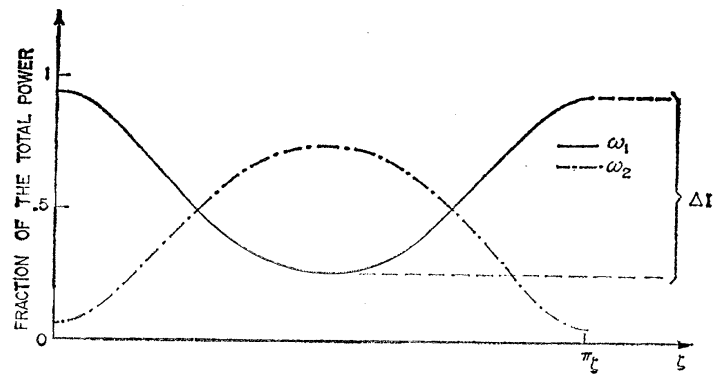

Frg. 2. Variation of the power in the fundamental and secondharmonic waves for arbitrary initial conditions. 
If the initial amplitude of the fundamental $\rho_{1}(0) \rightarrow 0$, then $\zeta_{0} \rightarrow-\infty$. The subharmonic $\rho_{1}$ is not generated from $\rho_{2}(0)$ in the ideal traveling wave geometry. The slightest amount of feedback will, however, start to generate the subharmonic from the noise level. This situation has been discussed by Kingston ${ }^{28}$.

The Eq. (5.12) can be used, if $\rho_{1}(0) \neq 0, \rho_{2}(0) \neq 0$, provided $\phi_{2}(0)-2 \phi_{1}(0)= \pm \pi / 2$. In the case of perfect matching this relative phase will be preserved.

Depending on whether $\sin \theta(0)= \pm 1$, either the second harmonic or the fundamental will be amplified first. If the fundamental gets amplified first $\left(\zeta_{0}<0\right)$, the second harmonic will decrease to zero, and then increase until all the power is in the second harmonic. If the second harmonic is amplified first $\left(\zeta_{0}>0\right)$, it goes directly to complete conversion.

If one defines

$$
y^{2}=\left(v^{2}-v_{a}^{2}\right) /\left(v_{b}^{2}-v_{a}^{2}\right),
$$

and labels the third root of the cubic equation $v^{2}\left(1-v^{2}\right)^{2}-\Gamma^{2}=0$ as $v_{c}^{2} \geq v_{b}^{2} \geq v_{a}^{2}$, Eq. (5.10) can be written

$$
\zeta=\frac{ \pm 1}{\left(v_{c}^{2}-v_{a}^{2}\right)^{1 / 2}} \int_{y(0)}^{y(\zeta)} \frac{d y}{\left[\left(1-y^{2}\right)\left(1-\gamma^{2} y^{2}\right)\right]^{1 / 2}}
$$

where

$$
\gamma^{2}=\left(v_{b}^{2}-v_{a}^{2}\right) /\left(v_{c}^{2}-v_{a}^{2}\right) .
$$

The quantity $y$ is, therefore, defined as a Jacobian elliptic function of $\zeta$. The normalized amplitudes $u$ and $v$ can now be written $\mathrm{as}^{29}$

$$
\begin{gathered}
v^{2}=v_{a}^{2}+\left(v_{b}^{2}-v_{a}^{2}\right) \operatorname{sn}^{2}\left[\left(v_{c}^{2}-v_{a}^{2}\right)^{1 / 2}\left(\zeta+\zeta_{0}\right), \gamma\right], \\
u^{2}=1-v_{a}^{2}-\left(v_{b}^{2}-v_{a}^{2}\right) \operatorname{sn}^{2}\left[\left(v_{c}^{2}-v_{a}^{2}\right)^{1 / 2}\left(\zeta+\zeta_{0}\right), \gamma\right],
\end{gathered}
$$

where $\zeta_{0}$ is determined by the initial condition [i.e., $y(0)]$ and the value of $\gamma$.

The results of this analysis are summarized in Fig. 4. The area bounded by the curves for $u^{2}$ and $v^{2}$ show the interval of variation of these quantities as a function of the initial condition $\Gamma^{2}$. Note that Eqs. (5.7) and (5.8) give a maximum value, $\Gamma_{\max }{ }^{2}=(4 / 27)=0.148$.

\section{B. Imperfect Phase Matching}

If the phase velocities of the fundamental and the second harmonic are not perfectly matched, $\Delta k \neq 0$ and thus $\Delta s \neq 0$, Eq. (5.5) can still be integrated by the method of the variation of the parameter in the solution of the homogeneous equation. One thus obtains the more general solution

$$
v u^{2} \cos \theta+\frac{1}{2} \Delta s v^{2}=\Gamma_{\Delta s}
$$

where $\Gamma_{\Delta s}$ can be expressed in terms of $\Gamma$ defined by Eq. (5.8)

$$
\Gamma_{\Delta s}=\Gamma+\frac{1}{2} \Delta s v^{2}(0) \text {. }
$$

${ }^{28}$ R. H. Kingston, Proc. IRE (Inst. Radio Engrs) 50, 472 (1962).

${ }^{29}$ E. T. Whittaker and G. N. Watson, A Course of Modern Analysis (Cambridge University Press, New York, 1927), 4th ed., p. 490.

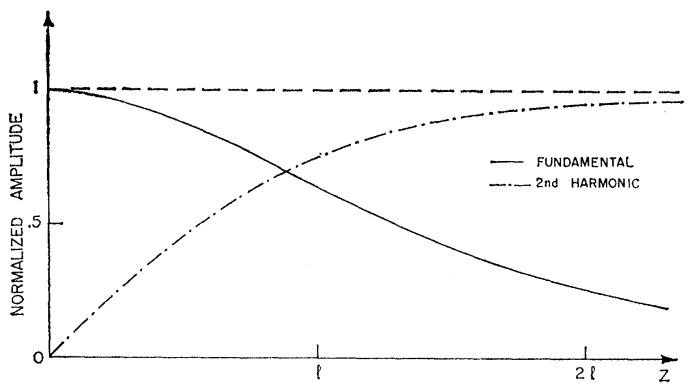

FIG. 3. The growth of the normalized second-harmonic amplitude and decrease of the normalized fundamental amplitude for perfect phase matching, if the second harmonic builds up from zero.

The generalization of Eq. (5.10) is

$\zeta= \pm \frac{1}{2} \int_{v^{2}(0)}^{v^{2}(\zeta)} \frac{d\left(v^{2}\right)}{\left[v^{2}\left(1-v^{2}\right)^{2}-\left\{\Gamma-\frac{1}{2} \Delta s\left[v^{2}-v^{2}(0)\right]\right\}^{2}\right]^{1 / 2}}$

Everything previously said about Eq. (5.10) and the solutions for $\Delta k=0$ can now be carried over to Eq. (5.17). The solutions for $\Delta k \neq 0$ are given by Eqs. (5.13) and (5.14) with the same definition of $y^{2}$. The three roots, $v_{c}^{2} \geq v_{b}^{2} \geq v_{a}^{2} \geq 0$ are now the roots of

$$
v^{2}\left(1-v^{2}\right)^{2}-\left\{\Gamma-\frac{1}{2} \Delta s\left[v^{2}-v(0)^{2}\right]\right\}^{2}=0 .
$$

For the important case of $\rho_{1}(0) \gg \rho_{2}(0)$ with $\Delta k / k_{1} \ll 1$, and $\cos ^{2} \alpha_{1}=\cos ^{2} \alpha_{2} \approx 1$, the following approximations are useful

and

$$
\Gamma \approx\left[\rho_{2}(0) / \rho_{1}(0)\right] \cos \left[\phi_{2}(0)-2 \phi_{1}(0)\right]
$$

$$
\Delta s \approx \frac{\Delta k}{\left[\left(2 \omega^{2} K / k_{1}\right) \rho_{1}(0)\right]} \approx \Delta k l,
$$

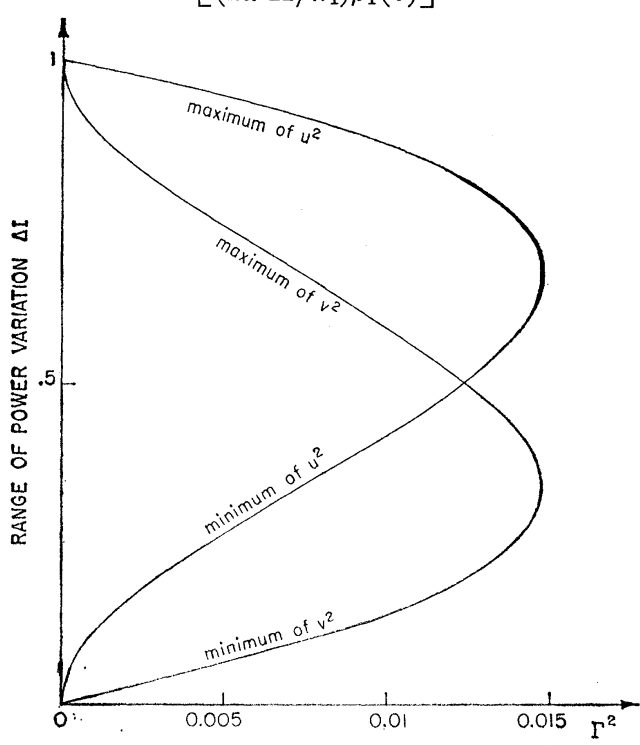

FIg. 4. The range of power variation in the fundamental and second-harmonic wave for perfect phase matching, as a function of the parameter $\Gamma^{2}$, defined in the text $[\mathrm{Eq} .(5.8)]$. 


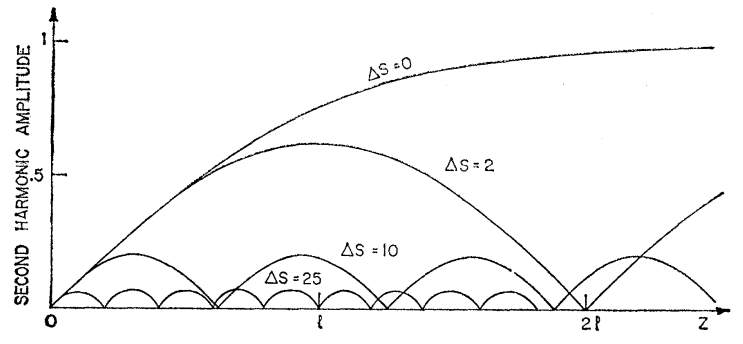

Fig. 5. The growth of the second-harmonic amplitude for varying degrees of phase mismatch.

where $l$ is defined by Eq. (5.13). The roots of Eq. (5.18) can, in general, be determined graphically. For the simple case of $\rho_{2}(0)=0[\Gamma=0$ and $v(0)=0]$

$$
\begin{aligned}
& v_{a}^{2}=0 \\
& v_{b}^{2}=\frac{1}{v_{c}^{2}}=\left[\frac{1}{\Delta s / 4+\left[1+(\Delta s / 4)^{2}\right]^{1 / 2}}\right]^{2}
\end{aligned}
$$

and the period is given by the elliptic integral

$$
\Pi_{\zeta}=\frac{2}{v_{c}} \int_{0}^{\pi / 2} \frac{d t}{\left[1-\left(v_{b} / v_{c}\right)^{2} \sin ^{2} t\right]^{1 / 2}} .
$$

If the condition for phase matching is nearly satisfied,

$$
\Delta s \approx \Delta k l \ll 1 \text {. }
$$

$v_{b}$ and $\Pi_{\zeta}$, given by Eqs. (5.21) and (5.22), are relatively insensitive to $\Delta s$, and substantial power conversion is obtained. Examples for several degrees of phase mismatching are shown in Fig. 5.

If $\Delta s \gg 1$, the approximation to the exact solution Eq. (5.17) can best be obtained by returning to Eq. (5.1). The most important experimental situation is imperfect matching, in which $\rho_{2}$ always remains small. In this case $\rho_{1}$ can be regarded as a constant of the motion.

If the second harmonic is initially zero, the approximate solutions to Eqs. (5.1b) and (5.1c) are

$$
\begin{aligned}
\theta & =\Delta k z / 2+\pi / 2, \\
\rho_{2}(z) & =\left[4 \rho_{1}(0) / \Delta k l\right] \sin (\Delta k z / 2) .
\end{aligned}
$$

Note that the phase advances as $\Delta k z / 2$ rather than $\Delta k z$. The phase velocity of the second harmonic, initially generated by the nonlinear interaction, is not the same as the velocity of the usual wave at $2 \omega$. If, however, the second harmonic is initially large enough so that $\Delta k \rho_{2}(0) \gg \rho_{1}(0) / l$, its phase velocity is the usual one for a wave at $2 \omega$.

$$
\begin{aligned}
\theta & =\Delta k z+\theta_{0}+\frac{1}{2} \pi, \\
\rho_{2}(z) & =\rho_{2}(0)+(2 / \Delta k l) \rho_{1}(0) \\
& \times\left[\sin \left(\theta_{0}+\Delta k z\right)-\sin \theta_{0}\right] .
\end{aligned}
$$

The power, $\rho_{2}{ }^{2}(z)$, has the period $1 / \Delta k$ regardless of $\rho_{2}(0)$. For $0<\theta<\pi$, power is transferred from $\omega$ to $2 \omega$. If $z$ is advanced by $\pi / \Delta k$, power is transferred back from $2 \omega$ to $\omega$. In neither case does the power in the second harmonic become large. The justification for the omission of higher harmonics in the coupled amplitude equations is, in fact, based on this observation. In general, they will have a more severe mismatch than the approximately matched phase velocities of the fundamental and second-harmonic wave. Consequently, they will only build up to a much smaller amplitude than the second harmonic.

\section{Lossy Medium}

Attenuation, or loss, can of course be incorporated into Maxwell's Eqs. (4.1) and (4.2), and into the wave Eq. (4.7) in a manner which is customary for a linear medium. In harmonic conversion, even very slight losses are intolerable, but it is of some interest to treat the case where the absorption length is comparable to or smaller than the interaction length $l$, defined previously.

The amplitude Eqs. (5.1) have to be replaced in a lossy dielectric by

$$
\begin{aligned}
d \rho_{1} / d z+b_{1} \rho_{1} & =-\left(2 \omega^{2} K / k_{1} \cos ^{2} \alpha_{1}\right) \rho_{1} \rho_{2} \sin \theta \\
d \rho_{2} / d z+b_{2} \rho_{2} & =\left(4 \omega^{2} K / k_{2} \cos ^{2} \alpha_{2}\right) \rho_{1}^{2} \sin \theta \\
d \theta / d z & =(\cos \theta / \sin \theta)(d / d z) \ln \left(\rho_{1}^{2} \rho_{2}\right)
\end{aligned}
$$

An exact solution can be found in the special case, $b_{1}=b_{2}=b$, by the substitutions,

$$
\begin{aligned}
y & =(1 / b)\left(1-e^{-b z}\right), \\
R_{1} & =\rho_{1} e^{b z}, \\
R_{2} & =\rho_{2} e^{b z} .
\end{aligned}
$$

For $\Delta k=0$ one obtains

$$
\begin{aligned}
d R_{1} / d y & =-\left(2 \omega^{2} K / k_{1} \cos ^{2} \alpha_{1}\right) R_{1} R_{2} \sin \theta, \\
d R_{2} / d y & =\left(4 \omega^{2} K / k_{2} \cos ^{2} \alpha_{2}\right) R_{1}^{2} \sin \theta, \\
d \theta / d y & =(\cos \theta / \sin \theta)(d / d y) \ln \left(R_{1}^{2} R_{2}\right) .
\end{aligned}
$$

The solution now proceeds as for the lossless case. For a significant power conversion it is necessary that $b \ll l^{-1}$.

\section{THREE COUPLED WAVES : EXACT SOLUTION}

The general amplitude Eqs. (4.9) can be written in their real and imaginary parts in a manner analogous to Eq. (5.1) for the second harmonics.

$d \rho_{1} / d z=-\left(\omega_{1}^{2} K / k_{1} \cos ^{2} \alpha_{1}\right) \rho_{2} \rho_{3} \sin \theta$, $d \rho_{2} / d z=-\left(\omega_{2}^{2} K / k_{2} \cos ^{2} \alpha_{2}\right) \rho_{3} \rho_{1} \sin \theta$, $d \rho_{3} / d z=\left(\omega_{3}^{2} K / k_{3} \cos ^{2} \alpha_{3}\right) \rho_{1} \rho_{2} \sin \theta$,

$$
\begin{array}{r}
\frac{d \theta}{d z}=\Delta k+K\left(\frac{\omega_{3}^{2}}{k_{3} \cos ^{2} \alpha_{3}} \frac{\rho_{1} \rho_{2}}{\rho_{3}}-\frac{\omega_{2}^{2}}{k_{2} \cos ^{2} \alpha_{2}} \frac{\rho_{3} \rho_{1}}{\rho_{2}}\right. \\
\left.-\frac{\omega_{1}^{2}}{k_{1} \cos ^{2} \alpha_{1}} \frac{\rho_{2} \rho_{3}}{\rho_{1}}\right) \cos \theta,
\end{array}
$$

where $\theta=\Delta k z+\phi_{3}(z)-\phi_{2}(z)-\phi_{1}(z)$ and $\Delta k=k_{3}-k_{2}-k_{1}$. 
The power flow per unit area parallel to the direction of propagation is

$$
W=\frac{c^{2}}{8 \pi}\left[\frac{k_{1} \cos ^{2} \alpha_{1}}{\omega_{1}} \rho_{1}{ }^{2}+\frac{k_{2} \cos ^{2} \alpha_{2}}{\omega_{2}} \rho_{2}{ }^{2}+\frac{k_{3} \cos ^{2} \alpha_{3}}{\omega_{3}} \rho_{3}^{3}\right] .
$$

The following substitutions greatly simplify the algebra.

$$
\begin{aligned}
& u_{1}=\left(\frac{c^{2} k_{1} \cos ^{2} \alpha_{1}}{8 \pi \omega_{1}^{2} W}\right)^{1 / 2} \rho_{1}, \\
& u_{2}=\left(\frac{c^{2} k_{2} \cos ^{2} \alpha_{2}}{8 \pi \omega_{2}{ }^{2} W}\right)^{1 / 2} \rho_{2}, \\
& u_{3}=\left(\frac{c^{2} k_{3} \cos ^{2} \alpha_{3}}{8 \pi \omega_{3}{ }^{2} W}\right)^{1 / 2} \rho_{3}, \\
& \zeta=K\left(\frac{8 \pi W}{c^{2}}\right)^{1 / 2}\left[\frac{\omega_{1}^{2} \omega_{2}^{2} \omega_{3}{ }^{2}}{k_{1} k_{2} k_{3} \cos ^{2} \alpha_{1} \cos ^{2} \alpha_{2} \cos ^{2} \alpha_{3}}\right]^{1 / 2} z .
\end{aligned}
$$

Equations (6.1) and (6.2) become

$$
\begin{aligned}
d u_{1} / d \zeta & =-u_{2} u_{3} \sin \theta \\
d u_{2} / d \zeta & =-u_{3} u_{1} \sin \theta \\
d u_{3} / d \zeta & =u_{1} u_{2} \sin \theta \\
d \theta / d \zeta & =\Delta S+K \cot \theta(d / d \zeta) \ln \left(u_{1} u_{2} u_{3}\right)
\end{aligned}
$$

where $\Delta S=\Delta k z / \zeta$.

The conservation of power flow is expressed by

$$
\omega_{1} u_{1}^{2}+\omega_{2} u_{2}^{2}+\omega_{3} u_{3}^{2}=1
$$

We can define three other constants $m_{1}, m_{2}, m_{3}$ :

$$
\begin{aligned}
& m_{1}=u_{2}^{2}+u_{3}{ }^{2}, \\
& m_{2}=u_{3}^{2}+u_{1}^{2}, \\
& m_{3}=u_{1}^{2}-u_{2}^{2},
\end{aligned}
$$

which constitute the vectorial Manley-Rowe relations taken in the direction of propagation.

The last of Eq. (6.4) can immediately be integrated to

$$
\cos \theta=\left(\Gamma+\frac{1}{2} \Delta \mathrm{Su}_{3}{ }^{2}\right) / u_{1} u_{2} u_{3} .
$$

Using Eq. (6.7) to eliminate $\sin \theta$ in Eq. (6.4), we get

$$
(d / d \zeta)\left(u_{3}^{2}\right)= \pm 2\left[\left(u_{1} u_{2} u_{3}\right)^{2}-\left(\Gamma+\frac{1}{2} \Delta S u_{3}\right)^{2}\right]^{1 / 2} .
$$

Using the Manley-Rowe relations, Eq. (6.6), we get

$\zeta= \pm \frac{1}{2} \int_{u_{3}{ }^{2}(0)}^{u_{3}{ }^{2}(\zeta)} \frac{d\left(u_{3}{ }^{2}\right)}{\left[u_{3}{ }^{2}\left(m_{2}-u_{3}{ }^{2}\right)\left(m_{1}-u_{3}{ }^{2}\right)-\left(\Gamma+\frac{1}{2} \Delta S u_{3}{ }^{2}\right)^{2}\right]^{1 / 2}}$.

The equation

$$
u_{3}{ }^{2}\left(m_{2}-u_{3}^{2}\right)\left(m_{1}-u_{3}^{2}\right)-\left(\Gamma+\frac{1}{2} \Delta S u_{3}{ }^{2}\right)^{2}=0
$$

has three roots for $u_{3}{ }^{2}$; call them $u_{3 c}{ }^{2} \geq u_{3 b}{ }^{2} \geq u_{3 a}{ }^{2} \geq 0$.

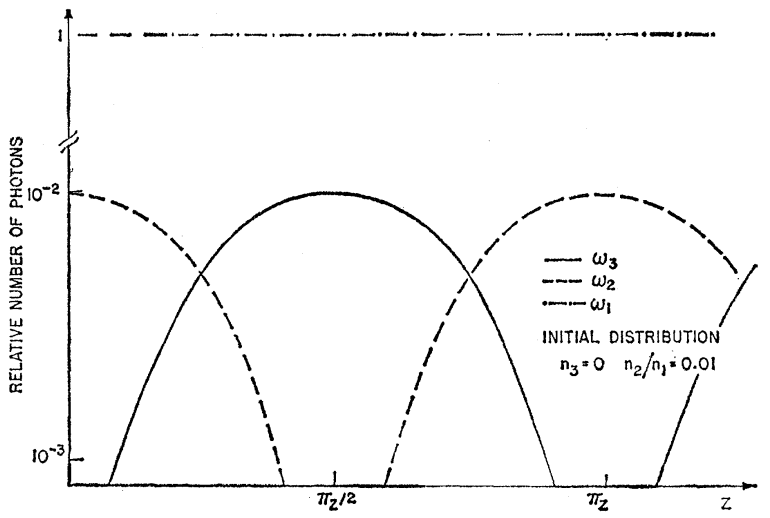

Fig. 6. Relative number of photons, as a function of $z$, in three traveling waves with perfect phase matching, $\omega_{3}=\omega_{2}+\omega_{1}$, $k_{3}=k_{2}+k_{1}$. The wave at $\omega_{1}$ has a very large number of quanta and may be considered as the pump in a parametric converter. (Up from $\omega_{2}$ to $\omega_{3}$, or down from $\omega_{3}$ to $\omega_{2}$.) The initial distribution is $n_{3}=0, n_{1}=100 n_{2}$.

The introduction of a quantity

$$
y^{2}=\left(u_{3}{ }^{2}-u_{3 a^{2}}{ }^{2}\right) /\left(u_{3 b}{ }^{2}-u_{3 a^{2}}\right)
$$

leads to a Jacobi elliptic integral in standard form and Eq. (6.9) can be written

$$
\zeta=\frac{ \pm 1}{\left(u_{3 c^{2}}{ }^{2}-u_{3 a^{2}}\right)^{1 / 2}} \int_{y(0)}^{y} \frac{d y}{\left[\left(1-y^{2}\right)\left(1-\gamma^{2} y^{2}\right)\right]^{1 / 2}},
$$

where

$$
\gamma^{2}=\left(u_{3 b^{2}}{ }^{2}-u_{3 a}{ }^{2}\right) /\left(u_{3 c}{ }^{2}-u_{3 a^{2}}{ }^{2} .\right.
$$

The general solution for the intensity of three waves is

$$
\begin{aligned}
& u_{3}{ }^{2}(\zeta)=u_{3 a^{2}}{ }^{2}+\left(u_{3 b}{ }^{2}-u_{3}{ }^{2}\right) \operatorname{sn}^{2}\left[\left(u_{3 c}{ }^{2}-u_{3 a}{ }^{2}\right)^{1 / 2}\left(\zeta+\zeta_{0}\right), \gamma\right] \text {, } \\
& u_{2}{ }^{2}(\zeta)=u_{2}{ }^{2}(0)+u_{3}{ }^{2}(0)-u_{3 a}{ }^{2}-\left(u_{3 b}{ }^{2}-u_{3 a}{ }^{2}\right) \\
& \times \operatorname{sn}^{2}\left[\left(u_{3 c}{ }^{2}-u_{3 a}{ }^{2}\right)^{1 / 2}\left(\zeta+\zeta_{0}\right), \gamma\right] \text {, } \\
& u_{1}^{2}(\zeta)=u_{1}^{2}(0)+u_{3}^{2}(0)-u_{3 a}{ }^{2}-\left(u_{3 b^{2}}{ }^{2}-u_{3 a^{2}}{ }^{2}\right) \\
& \times \operatorname{sn}^{2}\left[\left(u_{3 c}{ }^{2}-u_{3 a}{ }^{2}\right)^{1 / 2}\left(\zeta+\zeta_{0}\right), \gamma\right] \text {. }
\end{aligned}
$$

\section{A. Perfect Matching, $\Delta k=0$}

The simplest case of physical interest occurs when $\rho_{1}(0) \gg \rho_{2}(0)>0$ and $\rho_{3}(0)=0$. This corresponds to one very strong pump at $\omega_{1}$, converting a signal from frequency $\omega_{2}$ to $\omega_{3}$. In terms of the quantities occurring in Eqs. (6.12) and (6.13), this case is described by

$$
u_{3 c}{ }^{2}=m_{1} \gg m_{2}=u_{3 b}{ }^{2}, \quad u_{3 a}{ }^{2}=0, \quad \gamma^{2} \ll 1 .
$$

Equation (6.12) can be integrated directly

$$
u_{3}(\zeta)=m_{2}^{1 / 2} \sin \left(m_{1}^{1 / 2} \zeta\right) \text {. }
$$

If one sets $\cos ^{2} \alpha_{1}=\cos ^{2} \alpha_{2}=\cos ^{2} \alpha_{3}=1$, the amplitude of the electric field becomes

$$
\rho_{3}(z)=\left(\omega_{3} / \omega_{2}\right)^{1 / 2} \rho_{2}(0) \sin (\pi z / l),
$$

where the interaction length $l$ for the process is given by

$$
l^{-1}=\pi^{-1} K \omega_{2} \omega_{3}\left(k_{2} k_{3}\right)^{-1 / 2} \rho_{1}(0) .
$$




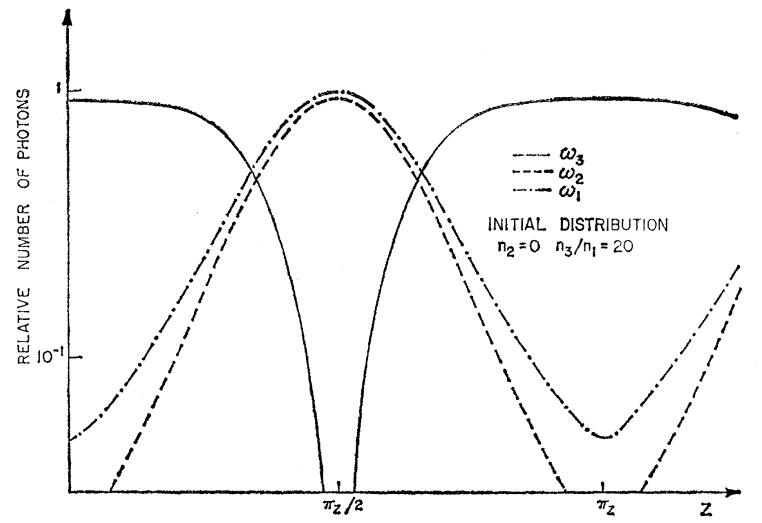

FIG. 7. Relative number of photons, as a function of $z$, in three traveling waves with perfect phase matching, $\omega_{3}=\omega_{2}+\omega_{1}$ $k_{3}=k_{2}+k_{1}$. The wave at $\omega_{3}$ has a very large number of quanta initially, and may be considered as the pump in a parametric amplifier. The initial distribution $n_{2}=0, n_{3}=20 n_{1}$

The period of the variation of energy is, in this case,

$$
\Pi_{z}=l \text {. }
$$

A typical solution is represented in Fig. 6 .

For $\omega_{3} \gg \omega_{2}$ there is power gain in the conversion of a photon to a higher frequency. The period, in space, for maximum gain is inversely proportional to the amplitude of the pump signal at $\omega_{1}$. This is the case usually encountered in parametric converters.

If the initial condition is such that there are initially equal numbers of photons at $\omega_{1}$ and $\omega_{2}$ and none at $\omega_{3}$, the solution becomes very simple. These initial conditions are equivalent to $m_{1}=m_{2}, m_{3}=0, \Gamma=0$. The period of the interaction is infinite; the photons at $\omega_{1}$ and $\omega_{2}$ become depleted at the same rate

$$
\begin{aligned}
& u_{1}=u_{2}=u_{1}(0) \operatorname{sech}(z / l), \\
& u_{3}=u_{1}(0) \tanh (z / l) .
\end{aligned}
$$

This special case is, therefore, similar to secondharmonic generation with perfect matching.

If $m_{1} \neq m_{2}$, the energy is transferred between the waves with a period in $\zeta$ [compare Eq. (5.11) and Eq. (6.9)], given by

$$
\Pi_{\zeta}=2 \int_{0}^{\left(m_{1}\right) 1 / 2} \frac{d u_{3}}{\left[\left(m_{2}-u_{3}^{2}\right)\left(m_{1}-u_{3}^{2}\right)\right]^{1 / 2}} .
$$

When the number of photons is depleted in one of the waves, the energy transfer reverses.

Consider next the case that a very large number is initially present at the highest frequency, $\omega_{3}$, a much smaller number at $\omega_{1}$, and none at $\omega_{2}$, i.e., $u_{3}(0) \gg u_{1}(0)$, $u_{2}(0)=0$. The Manley-Rowe relations [Eq. (6.6)] become

$$
\begin{aligned}
& m_{1}=u_{3}^{2}(0)+u_{1}^{2}(0), \\
& m_{2}=u_{3}^{2}(0) \\
& m_{3}=u_{1}^{2}(0)
\end{aligned}
$$

And thus,

$$
u_{3 c^{2}}{ }^{2}=u_{3}^{2}(0)+u_{1}^{2}(0), \quad u_{3 b^{2}}{ }^{2}=u_{3}^{2}(0), \quad u_{3 a^{2}}=0 .
$$

The amplified signal at $\omega_{1}$ given by Eq. (6.13) is

$$
\begin{aligned}
& u_{1}^{2}(\zeta)=u_{1}^{2}(0)+u_{3}^{2}(0) \\
& \times\left\{1-\operatorname{sn}^{2}\left[u_{3}(0)\left(\zeta-\zeta_{0}\right),\left(\frac{u_{3}^{2}(0)}{u_{3}^{2}(0)+u_{1}^{2}(0)}\right)^{1 / 2}\right]\right\} .
\end{aligned}
$$

If we define a length $l$,

$$
l^{-1}=K \omega_{1} \omega_{2}\left(k_{1} k_{2}\right)^{-1 / 2} \rho_{3}(0),
$$

then Eq. (6.17) can be rewritten

$$
\begin{aligned}
\rho_{1}^{2}(z)=\rho_{1}^{2}(0) & +\frac{\omega_{2}}{\omega_{3}} \rho_{3}^{2}(0) \\
\times & {\left[1-\operatorname{sn}^{2}\left(\frac{z-z_{0}}{l}, 1-\frac{1 \omega_{3}}{2} \frac{\rho_{1}^{2}(0)}{\omega_{1} \rho_{3}{ }^{2}(0)}\right)\right], }
\end{aligned}
$$

where $\rho_{3}(0) \gg \rho_{1}(0)$ has been used. An example of this case is shown in Fig. 7.

To satisfy the initial condition $\rho_{2}(0)=0, z_{0} / l$ must be set equal to $\frac{1}{2}$ period of the sn function.

For $\rho_{3}(0) \gg \rho_{1}(0)$ a half period can be approximated by ${ }^{30}$

$$
z_{0} / l=\frac{1}{2} \ln \left[16 \omega_{1} \rho_{3}^{2}(0) / \omega_{3} \rho_{1}^{2}(0)\right] .
$$

Thus, the maximum possible power transferred to the signal at $\omega_{1}$ occurs in a length $z_{0}$,

$$
\begin{aligned}
z_{0}=(K / 2) \omega_{1} \omega_{2}\left(k_{1} k_{2}\right)^{-1 / 2} \rho_{3}(0) & \\
& \times \ln \left[16 \omega_{1} \rho_{3}{ }^{2}(0) / \omega_{3} \rho_{1}{ }^{2}(0)\right],
\end{aligned}
$$

and the period of the variation of energy is

$$
\Pi_{z}=2 z_{0} \text {. }
$$

It is instructive to compare the length $z_{0}$ for appreciable power transfer from $\omega_{3}$ to $\omega_{1}$ to the length necessary for significant second-harmonic generation at $2 \omega_{3}$. That this last length is approximately the same as $l$ defined in Eq. (6.18) can be seen by comparison with Eq. (5.13). Equation (6.20) is thus a direct measure of the difficulty in making a frequency converter relative to the difficulty in construction of a second-harmonic generator. If $16 \omega_{1} \rho_{3}{ }^{2}(0) / \omega_{3} \rho_{1}{ }^{2}(0)=10^{6}$, $z_{0} / l$ is approximately 7.5 . Frequency conversion should not be appreciably more difficult than second-harmonic generation.

Equation (6.17) also describes parametric amplification in the case where the pump is allowed to be depleted. One can, for example, use this solution to estimate the extent to which the amplification is nonlinear.

${ }^{30}$ E. Jahnke, F. Emde, F. Lösch, Tables of Functions (McGrawHill Book Company, Inc., New York, 1960), 6th ed., p. 73. 
If the initial numbers of photons at $\omega_{3}$ and $\omega_{1}$ have the same order of magnitude, the energy transfer between the waves has again a typical oscillatory behavior. Reversal takes place when the number of quanta in one of the waves is depleted. Figure 8 shows the solution for the initial condition $u_{1}^{2}(0)=1.5 u_{3}^{2}(0)$, $u_{2}^{2}(0)=0$. In this case the period of the variation of energy is given by

$$
\Pi_{z}=2-\int_{\zeta}^{\left(m_{2}\right)^{1 / 2}} \frac{d u_{3}}{\left(m_{2}-u_{3}^{2}\right)^{1 / 2}\left(m_{1}-u_{3}^{2}\right)^{1 / 2}}
$$

where the ratio $z / \zeta$ has been defined in Eq. (6.3). These cases illustrate a generalization of the usual parametric amplifier theory.

\section{B. Imperfect Matching}

The general solution, Eq. (6.13), also describes the important experimental situation in which $\Delta k \neq 0$. In this case, the energy transfer between the waves reverses its trend when the relative phase between the waves has undergone a $180^{\circ}$ shift. The discussion for second-harmonic generation in the case of phase mismatch can be carried over step by step to the interaction between three waves. For severe mismatch, one obtains sinusoidal variations in amplitudes, similar to Eq. (5.24), which are well known in the conventional theory of traveling-wave parametric converters. The influence of the mismatch on the process $u_{3}(0) \gg u_{1}(0)$, $u_{2}(0)$ is represented in Fig. 9. The maximum number of photons which can be obtained at $\omega_{1}$ is limited by the degree of mismatch $\Delta S$.

\section{Lossy Medium}

The effects of losses can be introduced into Eq. (6.1) just as they were introduced in Sec. $V$ for the two-wave problem. The results are that the "decay length" must not be small compared to the characteristic length for

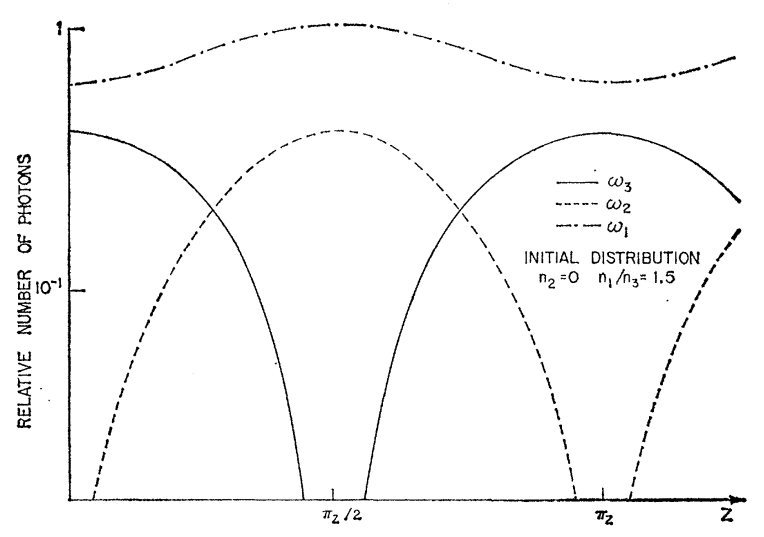

FIG. 8. Relative number of photons as a function of $z$ in three traveling waves with perfect phase matching. The waves at $\omega_{1}$ and $\omega_{3}$ have numbers of photons of the same order of magnitude. The initial distribution is $n_{2}=0, n_{1}=1.5 n_{3}$.

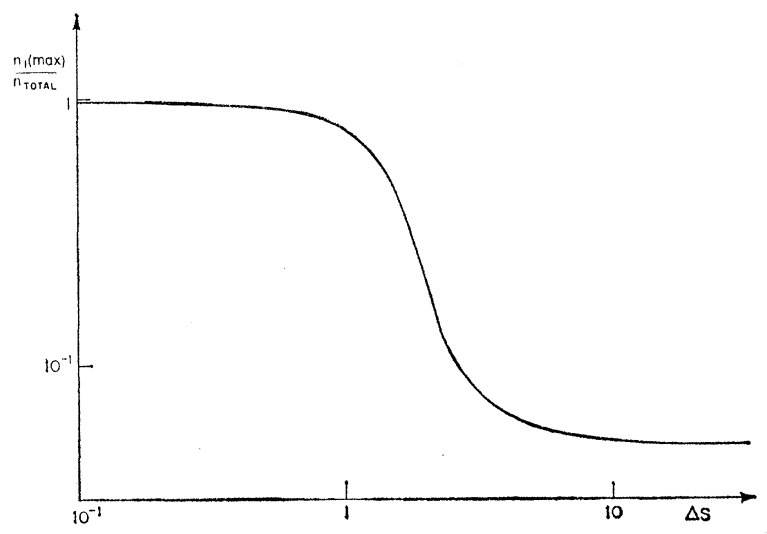

FIG. 9. The ratio of the maximum number of photons at $\omega_{1}$ $\left(n_{1} \max \right)$ to the total number of incident photons $[n(0)]$ as a function of $\Delta s$. [Initial distribution $n_{3}(0) / n_{1}(0)=20, n_{2}(0)=0$.]

interaction between frequencies if the parameter effects are to occur.

\section{FOUR INTERACTING WAVES-THIRD- HARMONIC GENERATION}

Equations (4.12) describing the general interaction of four waves and Eqs. (4.11) which are specialized to third-harmonic generation can be treated in the same way as the interaction of three waves and second harmonics were treated in the last two sections. It will suffice here to point out several salient features of the extension to higher-order interactions.

The real and imaginary parts of the equations for third-harmonic generation, Eq. (4.11), are

$$
\begin{aligned}
d \rho_{1} / d z= & -\left(3 \omega^{2} C \rho_{1}^{2} \rho_{3} / k_{1} \cos ^{2} \alpha_{1}\right) \sin \theta \\
d \rho_{3} / d z= & \left(9 \omega^{2} C \rho_{1}^{3} / k_{3} \cos ^{2} \alpha_{3}\right) \sin \theta \\
d \theta / d z= & \Delta k+(\cos \theta / \sin \theta)(d / d z) \ln \left(\rho_{3} \rho_{1}^{3}\right) \\
& +9 \omega^{2}\left(3 C^{\prime \prime} / k_{3} \cos ^{2} \alpha_{3}-C^{\prime} / k_{1} \cos ^{2} \alpha_{1}\right) \rho_{1}{ }^{2} \\
& +9 \omega^{2}\left(C^{\prime \prime \prime} / k_{3} \cos ^{2} \alpha_{3}-C^{\prime \prime} / k_{1} \cos ^{2} \alpha_{1}\right) \rho_{3}{ }^{2}
\end{aligned}
$$

The only effect from the terms in Eqs. (4.11) containing the constants $C^{\prime}, C^{\prime \prime}$, and $C^{\prime \prime \prime}$ is to change the phase velocities of the two waves at $\omega$ and $3 \omega$ from the small signal values. These arise because the time averages of $E_{1}{ }^{2}$ and $E_{3}{ }^{2}$ produce the usual dc Kerr effect proportional to the electric field squared. This is also true even if there is only one wave, say $\omega$, propagating in a nonlinear medium. For large enough signals the velocity depends on the amplitude of the wave.

If there is a mismatch in the phase velocities, so that $\Delta k$ is larger than either of the last two terms of Eq. (7.1c), one can assume the relative phase advances as

$$
d \theta / d z \approx \Delta k+\cot \theta(d / d z) \ln \left(\rho_{3} \rho_{1}^{3}\right)
$$

since $C$ has the same order of magnitude as $C^{\prime}, C^{\prime \prime}$, and $C^{\prime \prime \prime}, \rho_{1}$ never changes very much from its initial value and can be regarded as an approximate constant of the motion. The discussion now follows exactly as for 
second harmonics, Eqs. (5.24). If the third harmonic is initially generated by the nonlinear interaction, it does not have the same velocity as the usual wave at. $3 \omega$. The solutions are

$$
\begin{gathered}
\theta \approx \frac{\Delta k z}{2}+\frac{\pi}{2}, \\
\rho_{3}(z)=\frac{9 \omega^{2} C \rho_{1}{ }^{3}(0)}{k_{3} \cos ^{2} \alpha_{3}} \frac{\sin (\Delta k z / 2)}{(\Delta k / 2)} .
\end{gathered}
$$

If the initial value of $\rho_{3}$ is large enough, $\theta=\Delta k z+\pi / 2+\theta_{0}$ and $\rho_{3}$ is obtained by integration of Eq. (7.1b). The following substitutions,

$$
\begin{aligned}
& u=\left(\frac{c^{2}}{8 \pi W} \frac{k_{1}}{\omega_{1}} \cos ^{2} \alpha_{1}\right)^{1 / 2} \rho_{1}, \\
& v=\left(\frac{c^{2}}{8 \pi W} \frac{k_{3}}{\left(3 \omega_{1}\right)} \cos ^{2} \alpha_{3}\right)^{1 / 2} \rho_{3}, \\
& \zeta=C \frac{8 \pi W}{c^{2}} \frac{3 \omega_{1}\left(3 \omega_{1}^{4}\right)^{1 / 2}}{\left(k_{1}{ }^{3} k_{3} \cos ^{6} \alpha_{1} \cos ^{2} \alpha_{3}\right)^{1 / 2}} z,
\end{aligned}
$$

$$
\zeta= \pm \frac{1}{2} \int_{v^{2}(0)}^{v^{2}(\zeta)} \frac{d v^{2}}{\left[v^{2}\left(1-v^{2}\right)^{3}-\left[\Gamma+\frac{1}{4}(b-a)\left(1-v^{2}\right)^{2}+\frac{1}{2}(\Delta s+b) v^{2}\right]^{2}\right]^{1 / 2}} .
$$

For the third-harmonic power initially zero, $v(0)=0$ and $\Gamma=\frac{1}{4}(a-b)$. The solution for $v^{2}$ will oscillate between two roots of

$$
v^{2}\left\{\left(1-v^{2}\right)^{3}-v^{2}\left[\frac{1}{2}(\Delta s+a)+\frac{1}{4}(b-a) v^{2}\right]^{2}\right\}=0 .
$$

Equation (7.9) has for one root $v^{2}=0$, the lower limit for $v^{2}$. The next higher root will be less than unity, unless $a=b=\Delta s$. Physically, one cannot match the phase velocity of the two waves unless this equality holds. As $\rho_{1}{ }^{2}$ and $\rho_{3}{ }^{2}$ vary, the quadratic Kerr effect changes the phase velocities of the two waves. Without perfect phase matching, it is impossible to get complete conversion of power from $\omega$ to $3 \omega$. This is a rather academic point, however, since it only becomes important after a significant amount of harmonic generation has taken place. Any general interaction involving more than three waves will show this same effect.

\section{APPLICATIONS OF THE THEORY TO EXPERIMENTAL SITUATIONS}

\section{A. Harmonic and Subharmonic Generation}

The most studied nonlinear effect is, thus far, second-harmonic generation. The analysis of Sec. V shows that it is possible, in principle, to convert all incident power into the second harmonic. Since $\chi_{x y z} \mathrm{NL}$ is about $10^{-11}$ esu for $\mathrm{KDP},{ }^{14}$ the interaction length $l$ [Eq. (5.13)] has the order of magnitude of $10^{3} \mathrm{~cm}$ for an incident amplitude $\rho_{1}(0)=30 \mathrm{kV} / \mathrm{cm}$. For phase matching over a path $z=1 \mathrm{~cm}$, only about one part in where $W$ is the total power flow analogous to Eq. (5.2), reduce Eqs. (7.1) to

$$
\begin{aligned}
& d u / d z=-u^{2} v \sin \theta, \\
& d v / d z=u^{3} \sin \theta, \\
& d \theta / d z=\Delta s+\cot \theta(d / d z) \ln \left(v u^{3}\right)+a u^{2}+b v^{2},
\end{aligned}
$$

where $a, b, \Delta s$ can be obtained directly on making the substitutions. Equation (7.5c) can be integrated

$$
v u^{3} \cos \theta=\Gamma+(-a+b)\left(u^{4} / 4\right)+[(\Delta s+b) / 2] v^{2} .
$$

The conservation of energy equation is

$$
u^{2}+v^{2}=1
$$

Equations (7.6) and (7.7) substituted into Eq. (7.5b) lead to the integral equation

$10^{8}$ of the incident power is converted. It is possible to increase the peak power considerably, and field strengths of up to $10^{6} \mathrm{~V} / \mathrm{cm}$ may be attainable. Even then the required phase matching over the interaction length will certainly not be achieved for all rays in the solid angle of the beam. It will be necessary to apply phase correction schemes after the waves have traveled a distance $d=\pi(\Delta k)^{-1}$.

A phase shift of $180^{\circ}$ in $\theta=\Delta k z+2 \phi_{1}-\phi_{2}$ can be obtained in several ways. One can pass the waves through a dispersive linear dielectric phase correcting plate. It is more expedient from an experimental point of view to pass the light waves into another crystal of KDP, whose crystalline orientation is obtained by inversion of the first crystal, cf. Fig. 10. The sign of the third-order tensor, and therefore, of the interaction constant, is inverted in the inverted crystal. This can be repeated after each thickness $d$. An even simpler way to obtain the same result is to reflect both light waves after they have traversed a distance $d$. They each undergo an $180^{\circ}$ phase shift on reflection and, therefore, $\Delta \theta=2 \Delta \phi_{1}-\Delta \phi_{2}$ changes by $180^{\circ}$. In this case, the light waves, rather than the crystal, are inverted in space.

Another phase correcting scheme is to fold the path of one of the light rays into a resonant structure with a high-quality factor $Q$. Assume, e.g., that the second harmonic is reflected after a distance $d=\pi(\Delta k)^{-1}$, which corresponds to the spacing of the plates of a FabryPérot interferometer. After the second harmonic has traveled back to the front plate and is reflected again, 
it is exactly in phase once more with itself and the incident fundamental traveling wave. The effective interaction path is increased and conversion efficiency is increased by a factor $Q$. If the structure were made resonant simultaneously to both the fundamental and the second-harmonic frequency, the maximum size of the resonant structure is determined by $d=\pi Q^{-1}(\Delta k)^{-1}$. If both paths are folded, the structure is made smaller, but there is no gain in the maximum obtainable conversion. It is clear that very substantial improvements in the conversion factor could lead to a nearly complete conversion into the second harmonic. The maximum conversion reported thus far is $1: 10^{6}$.

Kingston ${ }^{28}$ has shown how the laser beam may pump a cavity tuned at a subharmonic frequency. In this case, the second harmonic feed power back into the fundamental. According to the classical Eq. (4.10), this process can not start to build up for $A_{1}=0$. The fundamental or subharmonic frequency starts from the zero-point vibrations, or from the spontaneous emission of two subharmonic quanta for the loss of one quantum in the laser beam. This buildup is similar to the buildup of a self-excited oscillator from the noise level. The classical complex amplitude Eqs. (4.9) to (4.12) are in a form which is readily subjected to quantization. The importance of zero-point vibrations and quantum noise has been discussed in a very interesting paper by Louisell, Yariv, and Siegman. ${ }^{31}$ Their technique could be applied to our equations.

\section{B. Frequency Conversion}

Similar considerations apply to the phase correction and the effective interaction length between three waves. An important application would be the generation of millimeter wave or far infrared radiation as the beat note at the difference frequency of two lasers. Two ruby lasers at different temperatures or two Zeeman components of a single laser in a strong magnetic field could provide such a beat. The Manly-Rowe relations show that, with a peak power of $10 \mathrm{MW}$ in a ruby laser pulse of about $10^{-8} \mathrm{sec}, 10 \mathrm{~kW}$ can be available at $0.7 \mathrm{~mm}$ during this pulse, if perfect phase matching is obtained. The coupling coefficient is proportional to $\chi_{x y z}\left(\omega_{m}=\omega_{3}-\omega_{2}\right)$. This quantity may differ appreciably from the corresponding quantity for doubling, because the ionic displacements play a larger role, if one of the frequencies is below the infrared absorption band.

An interesting situation arises if the objective is to detect a weak coherent light signal at $\omega_{s}$. In that case, one may use to advantage parametric up-conversion by means of a powerful laser beam at the pump frequency $\omega_{p}$. The output is taken at the sum frequency $\omega_{I}=\omega_{s}+\omega_{p}$ in an up-converter. If the phase velocity matching is complete, all available signal power is converted to

${ }^{31}$ W. H. Louisell, A. Yariv, and A. E. Siegman, Phys. Rev. 124, 1646 (1961).

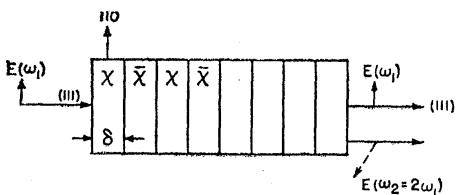

(a) $\mathrm{ZnS}$

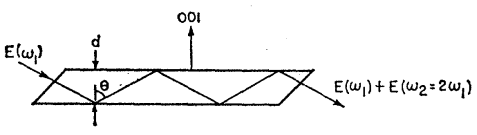

(b) Quartz

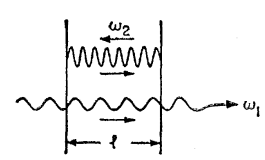

(c)

Fig. 10. Three experimental arrangements to provide phase correction, if the phase velocities of the fundamental and second harmonic are not perfectly matched. (a) After a distance $\delta$ $=\pi\left(k_{2}-2 k_{1}\right)^{-1}$ the crystal is replaced by its inversion image. The nonlinear susceptibility $\chi_{x y z}$ changes sign. The linear optical properties remain the same. This scheme can, of course, also be used in noncubic piezoelectric crystals. (b) Both fundamental and second harmonic undergo multiple total reflections in a crystal of thickness $d=\left(k_{2}-2 k_{1}\right)^{-1} \pi \cos \theta$. On each reflection $E_{1}$ and $E_{2}$ undergo a $180^{\circ}$ phase shift, the product $E_{2} E_{1}{ }^{2}$ changes sign. (c) The traveling wave at $\omega_{1}$ pumps the interferometer cavity, which contains a nonlinear dielectric and is resonant at $\omega_{2}, l=n \lambda_{2} /$ $2<\left(k_{2}-2 k_{1}\right)^{-1} \pi$. The backward harmonic wave does not interact with the pump. On each forward pass it has the correct phase for amplification.

power in a wave at $\omega_{I}$. This wave can be detected with excellent discrimination against the pump power at $\omega_{p}$. If $\omega_{s}$ is in the infrared and $\omega_{p}$ in the red, $\omega_{I}$ may be in the green or even blue and may be detected with high efficiency in a photomultiplier tube. This device is noiseless in the sense that in the absence of a signal, there would be no output at $\omega_{I}$. There is no spontaneous emission noise because the pump quanta cannot spontaneously create a quantum at $\omega_{I}$ without violating the conservation of energy. In this sense, such a device is better than a laser amplifier which always has an effective spontaneous emission noise temperature $\hbar \omega / k$. The effective interaction length for this situation is described by Eq. (6.16).

\section{C. dc and Microwave Kerr Effect}

The theory of the electro-optic Kerr effect was already developed in the early days of quantum mechanics. ${ }^{32} \mathrm{It}$ is contained in our theory as the special case that one of the frequencies is zero, $\omega_{3}=\omega_{2}=\omega$, $\omega_{1}=0$. Consider, for example, a much used geometry in KDP. The dc electric field $E_{\mathrm{do}}$ is applied along the tetragonal $z$ axis. A light wave linearly polarized along the $x$ axis propagates in the $z$ direction. The nonlinear coupling coefficient $\chi_{y x z}\left(\omega_{3}=\omega_{2}+0\right)$ will generate a wave at the same frequency and wave vector, linearly

${ }^{32} \mathrm{M}$. Born and P. Jordan, Elementare Quantenmechanik (Springer-Verlag, Berlin, 1930), p. 259. 
polarized in the $y$ direction. The coupled amplitude equations for this case may be written as [compare Eq. (5.1)],

$$
\begin{aligned}
& (d / d z) \rho_{x}(\omega)=K \rho_{y}(\omega) E_{\mathrm{dc}} \sin \left(\phi_{x}-\phi_{y}\right) \\
& (d / d z) \rho_{y}(\omega)=-K \rho_{x}(\omega) E_{\mathrm{de}} \sin \left(\phi_{x}-\phi_{y}\right) .
\end{aligned}
$$

They describe the polarization of the wave under the influence of the Kerr effect as it progresses along the $z$ direction. The more conventional description for this effect is to say that the uniaxial crystal has become optically biaxial by the application of the dc field. The normal modes of polarization for a wave propagating along the $z$ axis have a linear polarization along new axes, $x^{\prime}$ and $y^{\prime}$, which make angles of $45 \mathrm{deg}$ with the crystalline $x$ and $y$ axis. These modes have propagation constants which differ by an amount proportional to $E_{\text {dc. }}$

The description of the Kerr effect in the context of this paper becomes more interesting if the dc field is replaced by a microwave field. A traveling-wave version of a microwave light modulator has been proposed ${ }^{33}$, and the present theory, although not strictly applicable, has a bearing on this situation. In a wave guide it is possible for the microwave field to have a longitudinal component. It is also possible to choose the dimensions of the wave guide in such a manner that the phase velocity of the microwave in the guide, $E_{z m}$ $\sim E_{z} \cos \left(\omega_{m} t-k_{1} z\right)$, matches the phase velocity of the light wave $E_{x} \cos \left(\omega_{2} t-k_{2} z\right)$. The latter can, of course, still be regarded as a wave in free space.

The extension of the formalism from free waves to guided modes is straightforward. The microwave field in the light modulator can, however, without any question be regarded as a parameter, since the photon flux in the microwave is so overwhelmingly larger than the flux of light quanta. The coupling by the nonlinear susceptibility $\chi_{y z x}\left(\omega_{3}=\omega_{m}+\omega_{2}\right)$ leads to a side band of light at the sum frequency, polarized in the $y$ direction. There is, of course, also a wave at the difference frequency $\omega_{4}=-\omega_{1}+\omega_{2}$. The microwave field may, in turn, interact with these sidebands to produce additional sidebands. All these light waves are approximately phase matched, because they differ so little in frequency. The theory of this paper is then not applicable. The parametric theory of $\operatorname{Simon}^{34}$ may, however, be used, since the microwave field can be considered as a fixed parameter.

This case illustrates the meaning of dispersion in the nonlinear susceptibility. This quantity is defined for a frequency triple in the case of quadratic nonlinearities. The permutation symmetry relations show that the same constant which describes microwave modulation of light also describes the generation of the microwave

${ }^{33}$ P. S. Pershan and N. Bloembergen, Advances in Quantum Iilectronics, edited by J. R. Singer (Columbia University Press, New York, 1961), p. 187.

${ }^{34}$ J. C. Simon, Trans. IRE MTT 8, 18 (1960). as a beat between two light waves,

$$
\begin{aligned}
\chi_{z y x}\left(\omega_{m}=\omega_{3}-\omega_{2}\right)=\chi_{y x z}\left(\omega_{3}=\omega_{2}+\omega_{m}\right) & \\
& \neq \chi_{z y x}\left(\omega_{3}=\omega_{2}+\omega_{m}\right) .
\end{aligned}
$$

It is necessary, however, to specify the same direction for the microwave field in each case. Ionic linear and nonlinear motions are involved to the same extent in both processes. If all three frequencies are in the visible region of the spectrum, the value of $x$ will be appreciably different, because the ionic motions take no part at all in this case. These results are described in detail by the microscopic equations of Sec. II.

\section{CONCLUSION}

It is necessary to stress three assumptions which have been made throughout the treatment.

1. Only waves which are strictly monochromatic in frequency are considered; i.e., no allowance has been made for the effect of the finite linewidth of the interacting waves.

2. Only waves with perfectly defined propagation vectors are considered; i.e., no allowance has been made for the effects of the finite divergence of real light beams.

3 . It has been assumed that the dispersion of the medium makes it permissible to ignore completely all but the small number of waves whose phase velocities are well matched. That is, no investigation has been made of the effects of waves propagating in the medium with phase velocities which are not well matched. These waves will have a small, but nonzero, interaction with the waves considered in the theory.

It is interesting to note that the nonlinearity provides in principle a means of thermal contact between electromagnetic modes. A lossless nonlinear dielectric could take the place of the carbon speck introduced by Boltzmann to insure thermalization of the electromagnetic field enclosed in a cavity.

Finally, it should be stressed that the considerations given here are restricted to an infinite, homogeneous, nonlinear medium. The very interesting effects that occur at the boundary of a nonlinear dielectric will be treated in another paper.

The following conclusions summarize the theory presented here. The intrinsic nonlinear properties of electrons and ions bound in atoms, molecules, and dense media can be connected with the macroscopic properties of Maxwell's field quantities in nonlinear dielectrics. This permits, in turn, a detailed description of the coherent nonlinear scattering processes in terms of macroscopic, nonlinear susceptibilities. The interaction between coherent light waves leads to a rigorous solution which shows that it is possible for the idealized cases considered here to convert power completely from some frequencies to others. This solution embodies a generalization of parametric amplifier theory. It allows for very large signal and/or idler power, with 
concomitant depletion of the pump power. The ManleyRowe relations have been derived in a quite general manner. Since the nonlinear properties have been incorporated into Maxwell's equation, the theory can readily be applied to a wide variety of experimental situations where nonlinearities play a role.

\section{APPENDIX}

The local field at the site of the ith atom in the unit cell can be written as

$$
\mathbf{E}^{(i) l}=\mathbf{E}+\sum_{j} \mathbf{L}^{(i j)} \cdot\left(\mathbf{P}^{\mathrm{L}(j)}+\mathbf{P}^{\mathrm{NL}(j)}\right),
$$

and the polarizations of the $i$ th atom at frequency $\omega_{3}$ are related to the local fields at $\omega_{1}$ and $\omega_{2}$ (i.e., $\left.\omega_{3}=\omega_{1}+\omega_{2}\right)$ by

$$
\begin{aligned}
\mathbf{P}^{L(i)}\left(\omega_{3}\right) & =\boldsymbol{\alpha}^{(i)}\left(\omega_{3}\right) \cdot \mathbf{E}^{(i) l}\left(\omega_{3}\right) ; \\
\mathbf{P N L}^{(i)}\left(\omega_{3}\right) & =\boldsymbol{\beta}^{(i)}\left(\omega_{3}=\omega_{2}+\omega_{1}\right): \mathbf{E}^{(i) l}\left(\omega_{2}\right) \mathbf{E}^{(i) l}\left(\omega_{1}\right) .
\end{aligned}
$$

$\mathbf{L}^{(i j)}$ and $\boldsymbol{\alpha}^{(i)}(\omega)$ are each $3 \times 3$ matrices. From Eqs. (A1) and (A2) we can obtain

$\mathbf{P}_{i} \mathbf{L}(i)=\boldsymbol{\alpha}^{(i)} \cdot \mathbf{E}^{(i) l}=\boldsymbol{\alpha}^{(i)} \cdot \mathbf{E}+\sum_{j} \boldsymbol{\alpha}^{(i)} \cdot \mathbf{L}^{(i j)}$

$$
\cdot\left(\mathbf{P}^{\mathrm{L}(j)}+\mathbf{P}^{\mathrm{N}(j)}\right) \text {. }
$$

When an equation has only one frequency, we will drop the $\omega$ designation. We now define a $3 \times 3$ tensor

$$
\mathbf{M}^{(i j)}=\delta_{i j}-\boldsymbol{\alpha}^{(i)} \cdot \mathbf{L}^{(i j)}
$$

so that Eq. (A2') becomes

$$
\begin{aligned}
\sum_{j} \mathbf{M}^{(i j)} \cdot \mathbf{P}^{\mathrm{L}(j)}=\boldsymbol{\alpha}^{(i)} \cdot \mathbf{E}+\mathbf{P}^{\mathrm{NL}(i)} & \\
& -\sum_{j} \mathbf{M}^{(i j)} \cdot \mathbf{P}^{\mathrm{NL}(j)} .
\end{aligned}
$$

If we were to write $\mathbf{M}^{(i j)}$ in its component form, we could consider it to be a $3 N \times 3 N$ matrix, where $N$ is the number of atomic sites per unit cell. It would then be obvious that this "supermatrix" has an inverse. This allows us to define a new set of $3 \times 3$ matrices, $\mathbf{R}^{(i j)}$, such that

$$
\sum_{j} \mathbf{M}^{(i j)} \cdot \mathbf{R}^{(j k)}=\sum_{j} \mathbf{R}^{(i j)} \cdot \mathbf{M}^{(j k)}=\delta_{i k} .
$$

Equation (A2 ${ }^{\prime \prime}$ ) can thus be manipulated to give

$$
\mathbf{P}^{\mathrm{L}(k)}+\mathbf{P}^{\mathrm{NL}(k)}=\sum_{i} \mathbf{R}^{(k i)} \cdot \boldsymbol{\alpha}^{(i)} \cdot \mathbf{E}+\sum_{i} \mathbf{R}^{(k i)} \cdot \mathbf{P}^{\mathrm{NL}(i)} .
$$

Summing over all atoms in the unit cell, we obtain

$$
\begin{aligned}
\mathbf{P}=\mathbf{P}^{\mathrm{L}}+\mathbf{P}^{\mathrm{NL}}=\sum_{i k} \mathbf{R}^{(k i)} \cdot \boldsymbol{\alpha}^{(i)} \cdot \mathbf{E} \\
\\
+\sum_{i k} \mathbf{R}^{(k i)} \cdot \mathbf{P N L}^{(i)} .
\end{aligned}
$$

On inspection it becomes clear that

and that

$$
(\varepsilon-1) / 4 \pi=\sum_{i k} \mathbf{R}^{(k i)} \cdot \boldsymbol{\alpha}^{(i)}
$$

with

$$
\mathbf{D}\left(\omega_{3}\right)=\boldsymbol{\varepsilon}\left(\omega_{3}\right) \mathbf{E}\left(\omega_{3}\right)+4 \pi \mathbf{P}^{\mathrm{NLS}}\left(\omega_{3}\right)
$$

$$
\mathbf{P}^{\mathrm{NLS}}\left(\omega_{3}\right)=\sum_{i k} \mathbf{R}^{\left({ }_{k} i\right)}\left(\omega_{3}\right) \cdot \mathbf{P N L}^{\mathrm{N}(i)}\left(\omega_{3}\right) .
$$

Write $\mathbf{P N L}^{(i)}\left(\omega_{3}\right)$ as a function of the microscopic nonlinear polarizability tensors and the macroscopic Maxwell fields at $\omega_{1}$ and $\omega_{2}$. If $P^{\mathrm{NL}} \ll P^{\mathrm{L}}$ Eqs. (A1) and (A2) can be combined as

$$
\sum_{j}\left(\delta_{i j}-\mathbf{L}^{(i j)} \cdot \boldsymbol{\alpha}^{(j)}\right) \cdot \mathbf{E}^{(j) 1}=\mathbf{E} .
$$

Energy considerations require the symmetry of polarizability and Lorentz tensors. With

and

$$
\boldsymbol{\alpha}^{(i)}=\left(\boldsymbol{\alpha}^{(i)}\right)^{T}
$$

$$
\mathbf{L}^{(i j)}=\mathbf{L}^{(j i)}=\left(\mathbf{L}^{(j i)}\right)^{T},
$$

where $\mathbf{A}^{T}$ is the transpose of $\mathbf{A}, \mathbf{E q}$. (A8) can be written as

$$
\sum_{j}\left(\mathbf{M}^{(j i)}\right)^{T} \cdot \mathbf{E}^{(j) l}=\mathbf{E} .
$$

Taking the transpose of Eq. (A4), this can be converted to

$$
\mathbf{E}^{(k) 1}=\sum_{i}\left(\mathbf{R}^{(i k)}\right)^{T} \cdot \mathbf{E} .
$$

Combining Eqs. (A9) with Eqs. (A7) and (A2) we get

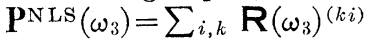

$$
\begin{array}{r}
\cdot \mathfrak{\Omega}^{(i)}\left(\omega_{3}=\omega_{2}+\omega_{1}\right): \sum_{j}\left[\left(\mathbf{R}^{(j i)}\left(\omega_{2}\right)\right)^{T} \cdot \mathbf{E}\left(\omega_{2}\right)\right] \\
\times \sum_{l}\left[\left(\mathbf{R}^{(l i)}\left(\omega_{1}\right)\right)^{T} \cdot \mathbf{E}\left(\omega_{1}\right)\right] .
\end{array}
$$

Define

$$
\mathbf{N}^{(i)}(\omega)=\sum_{l}\left(\mathbf{R}^{(l i)}(\omega)\right)^{T}
$$

and $P^{\mathrm{NLS}}$ can be written

$$
\mathbf{P}^{\mathrm{NLS}}\left(\omega_{3}\right)=\chi\left(\omega_{3}=\omega_{2}+\omega_{1}\right): \mathbf{E}\left(\omega_{2}\right) \mathbf{E}\left(\omega_{1}\right),
$$

where the $(a, b, c)$ component of $x$ is

$$
\chi_{a ; b, c}\left(\omega_{3}=\omega_{2}+\omega_{1}\right)=\sum_{i} \sum_{d, e, f} \beta_{d, e, f}{ }^{(i)}\left(\omega_{3}=\omega_{2}+\omega_{1}\right) N_{d, a}{ }^{(i)}\left(\omega_{3}\right) N_{e, b}{ }^{(i)}\left(\omega_{2}\right) N_{f, c}{ }^{(i)}\left(\omega_{1}\right) .
$$

The permutation symmetry relations are obvious. Equation (A12) can be written in tensor notation as

$$
\chi\left(\omega_{3}=\omega_{2}+\omega_{1}\right)=\sum_{i} \boldsymbol{\beta}^{(i)}\left(\omega_{3}=\omega_{2}+\omega_{1}\right): \mathbf{N}^{(i)}\left(\omega_{3}\right) \mathbf{N}^{(i)}\left(\omega_{2}\right) \mathbf{N}^{(i)}\left(\omega_{1}\right)
$$

